\section{Computable functionals}

by

\section{A. Grzegorczy k (Warszawa)}

Introduction, p. 168 .

\section{Table of contents}

\$ 1. Definition, p. 168

\$2. Basic properties, p. 172.

$\$ 3$. Uniformity Theorem, p. 180.

$\$$ 4. Applications to the computable analysis, p. 190.

References, p. 202

\section{Introduction}

In 1937 Banach and Mazur proposed a definition of computable functionals and computable real functions ${ }^{1}$ ). This definition is synthetic in a certain sense. On the other hand, it is very natural to give an inductive definition of computable functionals, analogical to the inductive definition of the class of computable (general recursive) functions in the arithmetic of integers. Such a definition is considered in this paper. I prove some general properties and indicate some applications to the analysis. An other notion of computable functional was introduced by Kleene in [5].

$\S 1$ contains the definitions and some general remarks, in $\S 2$ I prove some properties used in $\S \S 3$ and 4 . The aim of this paper is to prove the Uniformity Theorem contained in $\S 3$. In $\S 4$ I establish some consequences of the Uniformity Theorem in the computable analysis, $i . e$. the theorems stating that: the computable real functions are uniformly computable continuous in any segment, the maxima are computable numbers and the converse function to the one-one computable function is computable.

\section{Definition}

The notion of computable functionals will be introduced by means of an inductive definition. Let $\mathscr{N}$ be the set of natural numbers (non negative integers). Let $\widetilde{F}$ be the set of functions of one argument defined

1) See $\$ 2$, Property 8. Cf. Mazur [7]. over the set $\mathscr{N}$ and assuming the values of $\mathscr{N}$. We shall consider the functionals

$$
\Phi\left\langle f_{1}, \ldots, f_{n}\left(x_{1}, \ldots, x_{k}\right)=y\right.
$$

on $n$ arguments of the class $\mathfrak{F}$ and $k$ arguments of the class $\mathscr{N}$ assuming the values of the class $\mathscr{N}^{2}$ ). The class $\mathscr{K}$ of computable functionals is the least class of functionals of this type that satisfies the following conditions:

A. $\mathscr{K}$ contains as the initial functionals

1. the identity functional

2 . the constant functionals

$$
\mathbf{U}\langle f>(x)=f(x),
$$

$$
\begin{aligned}
& -f(x, y)=-(x, y)=x-y=\left\{\begin{array}{ccc}
x-y & \text { if } & x>y, \\
0 & \text { if } & x \leqslant y,
\end{array}\right. \\
& \mathbf{T} f(x, y)=\mathbf{T}(x, y)={ }^{y}, \\
& \mathbf{S}\langle f(x)=\mathbf{S}(x)=x+1 .
\end{aligned}
$$

B. $\mathscr{K}$ is closed under the following operations:

1. The operation of substitution. If $\Phi, \Psi \in \mathscr{K}$ and

$$
\begin{aligned}
& \chi \mathfrak{f}, y_{1}, \ldots, y_{t}\left(x_{1}, \ldots, x_{l-1}, \cdots_{l+1}, \ldots, x_{k}, y_{1}, \ldots, ?_{m}\right) \\
& =\Phi f\left(x_{1}, \ldots, x_{l-1}, \Psi\left\langle g_{1}, \ldots, c_{t}\left(y_{1}, \ldots, l_{m}^{\prime}\right), r_{l+1}, \ldots, r_{k}\right),\right.
\end{aligned}
$$

then $\chi \in \mathscr{K}$

2. The operation of identification of the variables representing numbers and representing functions. If e.g. $\Phi \in \mathscr{K}$ and

$$
\begin{gathered}
\Psi\left(x_{1}, \ldots, x_{k-1}\right)=\Phi\left\langle\left(x_{1}, \ldots, x_{j-1}, x_{j}, x_{j+1}, \ldots, x_{k-1}, x_{j}\right),\right. \\
\chi f_{1}, \ldots, f_{n-1}\left(x_{1}, \ldots, x_{k}\right)=\Phi\left\langle f_{1}, \ldots, f_{j}, \ldots, f_{n-1}, f_{j},\left(x_{1}, \ldots, x_{k}\right),\right.
\end{gathered}
$$

then $\Psi, \chi<\mathscr{K}$

3. The operation of effective minimum. If $\Phi \in \mathscr{K}$ and the following condition is satisfied:

$$
\prod_{f_{1}, \ldots, f_{n} \in \Re} \prod_{u_{1}, \ldots, u_{k} \in \mathfrak{N}} \sum_{x \in \mathfrak{N}} \Phi\left\langle f_{1}, \ldots, f_{n}\left(x, u_{1}, \ldots, v_{k}\right)=0\right.
$$

then to the class $\mathcal{K}$ belongs the functional $\Psi$ defined as follows:

$$
\Psi f_{1}, \ldots, f_{n}\left(u_{1}, \ldots, u_{k}\right)=(\mu x)\left[\Phi\left\langle f_{1}, \ldots, f_{n}\right)\left(x, u_{1}, \ldots, u_{k}\right)=0\right] .
$$

2) For the sake of formal convenience in the formulation of some theorems we shall often write the function-arguments in different parentheses $\langle>$ from those used for the number-arguments. Cf. properties 6 and 12 in $\$ 2$. To shorten the notation we shall often use gothic characters as abbreviations of complexes of variables: $f$ is an abreviation of $f_{1}, \ldots, f_{n}, x$ is an abreviation of $x_{1}, \ldots, x_{k}$, etc. 
where $\left(\mu_{x}\right)[A(f, \ldots, u, \ldots, x)]=\left\{\begin{array}{r}\text { the smalest } x \in \mathscr{N} \text { such that } A(f, \ldots, u, \ldots, x) \\ \text { if such an } x \text { exists } \\ 0 \text { if there are no such } x \text { that } A(f, \ldots, u, \ldots, x)\end{array}\right.$

The second possibility is excluded by condition (1). Hence condition (1) will be called the condition of effectiveness of the operation of minimum.

If we cancel in this definition the condition of effectiveness of the ninimum operation, we obtain the definition of the class of elementary definable functionals (see [2]). Hence all computable functionals are elementary definable.

Similarly by means of operations $1-3$ we can define the class of computable functionals of any higher type. The operation of identifying the variables must be extended to the higher types of variables.

The extension of the notion of computability to a higher type has several applications. We shall mention some of them.

a. The extension of the notion of recursive realizability to the higher types. S. C. Kleene and D. Nelson have introduced the concept of realizability of an elementary formula ${ }^{3}$ ). This concept can be extended to the set of all formulae of the simple theory of types. Hence some theorems concerning the intuitionistic elementary number theory can be extended to the intuitionistic number theory containing the simple theory of types.

b. The classification of the elementary definable sets of integers ean be extended to the sets of higher types ${ }^{4}$ ). The definitions are analogical. For example a set of functions $A$ is recursively denumerable if there exists a functional $\Phi \in \mathscr{K}$ such that

$$
f \in A \equiv \sum_{x \in \mathscr{Y}} \Phi\langle f\rangle(x)=0 .
$$

A set or a relation is computable if there exists a functional $\Phi \in \mathscr{K}$ such that

$$
A(f) \equiv \Phi\langle f\rangle=0 \quad \text { or } \quad A(f, g, x, y, \ldots) \equiv \Phi\langle f, g\rangle(x, y, \ldots)=0 .
$$

It is evident that the class of computable sets constitutes the Boolean field of sets:

$$
(a=0 \quad \text { and } \quad b=0) \equiv \mathbf{T}(a, \mathbf{T}(-(a, a), b)) \equiv a^{0^{b}}=0, \quad(a \neq 0) \equiv\left(0^{a}=0\right) .
$$

The classes of sets of higher degrees constitute rings of sets ${ }^{5}$ ). The proofs of all properties are the same as in the case of the sets of integers. $E$. $g$.

\footnotetext{
s) See Kleene [4] or [5] and Nelson [9].

4) See Kleene [3] and Mostowski [8].

5) Cf. the classification of Mostowski [8] and of Kleene [3]
}

if the set $A$ and its complement $-A$ are recursively denumerable, then the set $A$ is computable ${ }^{6}$ ). Indeed if

$$
\begin{aligned}
f \in A & \equiv \sum_{x \in \mathscr{V}} \Phi\langle f\rangle(x)=0, \\
f \in-A & \equiv \sum_{x \in \mathscr{N}} \Psi\langle f>(x)=0,
\end{aligned}
$$

$i$. e. the following condition is satisfied:

$$
\prod_{f \in \Im} \sum_{x \in \mathfrak{F}}(\Phi\langle f\rangle(x)=0 \vee \Psi\langle f\rangle(x)=0)
$$

(this is the condition of effectiveness of minimum), then the functional

$$
\chi\langle f\rangle=(\mu x)[\Phi\langle f\rangle(x)=0 \vee \Psi\langle f\rangle(x)=0]
$$

is computable and the set $A$ is also computable because

$$
f \in A \equiv \Phi\langle f\rangle(\chi\langle f\rangle)=0 .
$$

c. It is possible to define the subclasses of the class $\mathscr{K}$ in the same way as the well known subclasses of the class of computable functions. $E$. $g$. the class $\mathscr{R}$ of primitive recursive functionals is the smallest class of functionals that contains the same initial functionals as the class $\mathscr{K}$ and is closed under the operations: 1 . of substitution, 2. of identification of the function and number variables, 3 . of simple induction: i.e. if $\Phi, \Psi \in \mathscr{R}$ and

$$
\begin{aligned}
& \chi\langle\mathfrak{T}\rangle(0, y)=\Phi\langle\mathfrak{f}\rangle(y), \\
& \chi\langle\mathfrak{T}\rangle(x+1, y)=\Psi\langle\mathfrak{T}\rangle \mid x, y, \chi\langle\mathfrak{T}\rangle(x, y)),
\end{aligned}
$$

then $\chi \in \mathscr{R}$. The class $\mathcal{E}$ of elementary recursive functionals is the smallest class of functionals that contains the same initial functionals as the class $\mathscr{K}$ and is closed under the operations: 1 . of substitution, 2 . of identification of function and number variables, 3. of limited induction: i. e. if $\Phi, \Psi, \Omega \in \mathcal{E}$ and

$$
\begin{aligned}
& \chi\langle\mathfrak{T}\rangle(0, y)=\Phi\langle\mathfrak{f}\rangle(y), \\
& \chi\langle\tilde{f}\rangle(x+1, y)=\Psi\langle\mathfrak{f}\rangle \mid x, y, \chi\langle\tilde{f}\rangle(x, y)), \\
& \chi\langle\tilde{f}\rangle(x, y) \leqslant \Omega\langle\tilde{f}\rangle(x, y),
\end{aligned}
$$

then $\chi \in \mathcal{E}$. The class $\mathcal{E}^{0}$ of functionals is the smallest class of functionals that contains the same initial functionals as the class $\mathscr{K}$ excepting the functional $\mathbf{T}(x, y)=x^{y}$, and closed under the same operations as the class of elementary recursive functionals (cf. [1]).

${ }^{6}$ ) It is the theorem proved by Kleene for the set of integers (see [3]), and by Post [12]. 
It is evident that $\mathscr{E}^{\circ} \subset \mathcal{E} \subset \mathcal{R}$. The proof that $\mathscr{R} \subset \mathscr{K}$ is the same as for the computable functions. We shall show some properties of these classes in the sequel.

d. The applications to computable analysis will be considered in some detail in the following sections.

\section{§ 2. Basic properties}

1. For each computable function $f\left(x_{1}, \ldots, x_{k}\right)$ there exists a constant functional $\Phi \in \mathscr{K}$ equal to $f$, i. $e$.

$$
\prod_{g_{1}, \ldots, g_{n} \in \widetilde{F}} \Phi\left\langle g_{1}, \ldots, g_{n}\right\rangle\left(x_{1}, \ldots, x_{k}\right)=f\left(x_{1}, \ldots, x_{k}\right) .
$$

The class Com of computable functions is the least class of functions, that contains the functions $x-y, x^{y}, x+1$ and is closed under the same operations as the class $\mathscr{K}$ (cf. Robinson [13]).

2. The class $\mathscr{K}$ is closed under the scheme (2) of simple induction.

First we introduce the following computable functions and relations:

$$
\begin{aligned}
& x \leqslant y \equiv x-y=0, \\
& x=y \equiv x \leqslant y \wedge y \leqslant x, \\
& x \cdot y=(\mu z)\left[2^{z}=\left(2^{x, y}\right],\right. \\
& x+y=(\mu z)\left[2^{z}=2^{x} \cdot 2^{y}\right], \\
& {\left[\frac{x}{y}\right]=(\mu z)[y \cdot(z+1)>x \vee y=0],} \\
& x \mid y \equiv y \div x \cdot\left[\frac{y}{x}\right]=0,
\end{aligned}
$$

prime $(x) \equiv x \neq 0 \wedge x \neq 1 \wedge x=(\mu z)[x=0 \vee x=1 \vee(z>1 \wedge z \mid x)]$,

$\exp (x, y)=(\mu z)\left[y=1 \vee y=0 \vee \sim\left(y^{z+1} \mid x\right)\right]$,

$$
\begin{aligned}
\operatorname{Pri}(z)=(\mu y)[ & \sim(\operatorname{prime}(z)) \vee(y>1 \wedge \exp (y, 2)=2 \wedge z=(\mu v)[\text { prime }(v) \\
& \wedge(y<2 \vee \exp (y,(\mu u)[\operatorname{prime}(u) \wedge u>v]) \neq \exp (y, v)+1)])] .
\end{aligned}
$$

From the last definition it follows that

$$
\operatorname{Pri}(z)=\left\{\begin{array}{cl}
0 & \text { when } \sim(\text { prime }(z)), \\
2^{2} \cdot 3^{3} \cdot 5^{4} \cdot \ldots \cdot 2^{x+2} & \text { when } z=p_{x} \text { (the } x \text { th prime number) } ;
\end{array}\right.
$$

$$
p_{\boldsymbol{x}}=(\mu z)[\text { prime }(z) \wedge \exp (\operatorname{Pri}(z), z)=x+2]
$$

Now let us suppose that the functional $\chi$ is defined by means of scheme (2) of the preceding section, and $\Phi, \Psi \in \mathscr{K}$. Thus

$$
\begin{aligned}
\chi\langle\mathfrak{f}\rangle(x, y)=\exp ((\mu z)[ & \exp (z, 2)=\Phi\langle\tilde{\mathfrak{T}}\rangle(y) \\
\wedge x & \left.=(\mu u)\left[\exp \left(z, r_{u+1}\right) \neq \Psi\langle\mathfrak{T}\rangle\left(u, y, \exp \left(z, r_{u}\right)\right]\right], r_{x}\right)
\end{aligned}
$$

and therefore $\chi \in \mathscr{K}$. Hence the class $\mathscr{K}$ is closed under the operation of limited induction (3), and $\mathscr{R} \subset \mathscr{K}$.

3. The classes $\mathcal{E}^{\circ}, \mathcal{E}, \mathscr{R}, \mathscr{K}$ are closed under the operation of limited minimum. The relations of the classes $\mathcal{E}^{0}, \mathcal{E}, \mathscr{R}, \mathscr{K}$ are closed under the operations of propositional calculus and limited quantifiers.

The operation $(\mu x \leqslant z)[R\langle\mathfrak{f}\rangle(x, u)]=(\mu x)[x \leqslant z \wedge R\langle\mathfrak{f}\rangle(x, u)]$ of limited minimum can be defined by means of limited induction:

$(\mu x \leqslant 0)[R\langle\mathcal{T}\rangle(x, u)]=0$,

$$
\begin{aligned}
& (\mu x \leqslant z+1)[R\langle\mathfrak{f}\rangle(x, u)]=\left\{\begin{array}{r}
(\mu x \leqslant z)[R \ldots] \text { if }(\mu x \leqslant z)[R \ldots] \neq 0 \\
\text { or } R\langle\mathfrak{f}>(0, u) \text { or } \sim(R<\mathfrak{f}\rangle(z+1, u)), \\
z+1 \text { if } R\langle\mathfrak{f}\rangle(z+1, u) \text { and }(\mu x \leqslant z)[R \ldots]=0 \\
\text { and } \sim(R<\{>(0, u)),
\end{array}\right. \\
& (\mu x \leqslant z)[R\langle\mathfrak{f}\rangle(x, u)]<z+1 .
\end{aligned}
$$

The operation of limited quantifier can be expressed by means of limited minimum:

$$
\sum_{x \leqslant z} R(\ldots x \ldots) \equiv R(\ldots(\mu x \leqslant z)[R(\ldots x \ldots)] \ldots) .
$$

Let us now introduce some functions necessary in the sequel.

$$
\begin{array}{ll}
\mathrm{Pa}(x, y)=(x+y)^{2}+x, & {[\sqrt{x}]=(\mu z)\left[(z+1)^{2}>x\right],} \\
\mathrm{Fi} z=z-([\sqrt{z}])^{2}, & \text { Sc } z=[\sqrt{z}]-\mathrm{Fi} z .
\end{array}
$$

$\mathrm{Pa}, \mathrm{Fi}$, Se are pairing functions. I. e. they satisfy the conditions:

$$
\begin{array}{r}
\operatorname{Pa}(\mathrm{Fi} z, \mathrm{Se} z)=z, \quad \operatorname{Fi}(\mathrm{Pa}(x, y))=x, \quad \operatorname{Se}(\mathrm{Pa}(x, y))=y, \\
\max [a, b]=\left\{\begin{array}{lll}
a & \text { if } a \geqslant b, \\
b & \text { if } b \geqslant a,
\end{array}\right. \\
\operatorname{Max} \Phi\langle\mathfrak{f}\rangle(u, s) \text { for } s \leqslant x=(\mu y)\left[\sum_{s \leqslant x} y=\Phi\langle\mathfrak{f}\rangle(u, s)\right. \\
\left.\wedge \prod_{s \leqslant x} \Phi\langle\mathfrak{f}\rangle(u, s) \leqslant y\right] .
\end{array}
$$

4. Each functional $\Phi \in \mathscr{K}$ can be presented in the canonical form

$$
\Phi\langle\mathfrak{f}\rangle(u)=\mathrm{E}((\mu x)[\Psi\langle\mathfrak{f}\rangle(u, x)=0])
$$


where $\mathrm{E} x=\mathrm{Fi} x$ or $\mathrm{E} x$ is any other constant function that assumes any number infinitely many times, and $\Psi \in \mathcal{E}^{\text {) }}$ and $\Psi$ satisfies the condition of effectiveness ${ }^{7}$ ).

The proof is the same as in the case of computable functions.

э. If $\Phi \in \mathscr{K}$ and for given $\mathrm{g}, f$,

$$
\prod_{x_{1}, \ldots, x_{n} \in \mathscr{V N}} \Phi\langle\mathfrak{g}\rangle\left(x_{1}, \ldots, x_{n}\right)=f\left(x_{1}, \ldots, x_{n}\right),
$$

then $f$ is computable in $\mathrm{g}$.

A function $f$ is said to be computable in $g_{1}, \ldots, g_{k}$ if $f$ belongs to the smallest class of functions that contains the initial functions: $g_{1}, \ldots, g_{k}$, $x-y, x^{y}, x+1$, and is closed under the operations of substitution, of dentification of the number variables and of effective minimum (cf. e. g. Kleene [5]).

The proof of property 5 is inductive. If $\Phi$ is one of the initial functionals of the class $\mathscr{K}$ then $f_{\Phi}$ is the initial function of the class of functions computable in $g_{1}, \ldots, g_{k}$. If $\Psi$ and $\Omega$ possess property 5 and $\Phi$ is obtained from $\Psi$ and $\Omega$ by one of the operations $1,2,3$, then $f_{\Phi}$ is obtained in the class of functions computable in $g_{1}, \ldots, g_{k}$ by means of the same operation.

6. The class $\mathscr{K}$ is closed under the operation of functional substitution. I. e. if $\Phi, \Psi \in \mathscr{K}$ and

$$
\begin{gathered}
\Phi\langle\mathfrak{f}\rangle_{\mathfrak{x}}(z)=\Phi\langle\mathfrak{f}\rangle(\mathfrak{x}, z), \\
\chi\langle\mathfrak{f}, \mathfrak{g}\rangle(\mathfrak{x}, \mathfrak{y})=\Psi\left\langle\mathfrak{g}, \Phi\langle\mathfrak{f}\rangle_{\mathfrak{x}}\right\rangle(\mathfrak{y}),
\end{gathered}
$$

then $\chi \in \mathscr{K}$, and similarly if $\chi$ is obtained by means of the substitution of the function $\Phi\langle\mathfrak{T}\rangle_{\text {I }}$ for more than one function variable $g_{1}, \ldots, g_{n}$ in the functional $\Psi$, then $\chi \in \mathcal{K}$.

This stronger formulation is necessary for the sake of the following inductive proof. If $\chi$ is an initial functional, then it is interesting to verify the theorem when $\chi=U$ :

$$
U\left\langle\Phi\langle\mathfrak{f}\rangle_{\mathfrak{x}}\right\rangle(y)=\Phi\langle\mathfrak{f}\rangle_{\mathfrak{x}}(y)=\Phi\langle\mathfrak{f}\rangle(\mathfrak{x}, y)
$$

Now let us suppose that $\chi \in \mathscr{K}$ and that the equations (4) and (5) are satisfied. Let the functional $\Omega$ be obtained from $\Psi$ by substitution. E. g. $\sigma \in \mathscr{K}$ and

$$
\Omega\langle\mathfrak{h}, \mathfrak{g}, g\rangle(\mathfrak{y}, \mathfrak{u})=\sigma\langle\mathfrak{h}\rangle(\mathfrak{u}, \Psi\langle\mathfrak{g}, g\rangle(\mathfrak{y}))
$$

7) It is the analogue of Post-Markov Theorem. Cf. Grzegorczyk [1], Markov [6],
[5].
Hence (5) implies that

$$
\begin{gathered}
\Omega\left\langle\mathfrak{h}, \mathfrak{g}, \Phi\langle\mathfrak{f}\rangle_{\mathfrak{x}}\right\rangle(\mathfrak{l}, \mathfrak{u})=\sigma\langle\mathfrak{h}\rangle(\mathfrak{u}, \Psi\langle\mathfrak{g}, \Phi\langle\mathfrak{f}\rangle\rangle(\mathfrak{l})) \\
=\sigma\langle\mathfrak{h}\rangle(\mathfrak{u}, \chi\langle\mathfrak{f}, \mathfrak{g}\rangle(\mathfrak{x}, \mathfrak{l})) .
\end{gathered}
$$

Thus the functional substitution can be reduced to the number-argument substitution. In other cases of substitution the proof is similar.

If $\Omega$ is obtained from $\Psi$ by means of the minimum operation:

then, according to $(5)$,

$$
\Omega\langle\mathfrak{g}, g\rangle(\mathfrak{y})=(\mu z)[\Psi\langle\mathfrak{g}, g\rangle(\mathfrak{y}, z)=0],
$$

$$
\begin{gathered}
\Omega\left\langle\mathfrak{g}, \Phi\langle\mathfrak{f}\rangle_{\mathfrak{x}}\right\rangle(\mathfrak{l})=(\mu z)\left[\Psi\left\langle\mathfrak{g}, \Phi\langle\mathfrak{f}\rangle_{\mathfrak{x}}\right\rangle(\mathfrak{l}, z)=0\right] \\
=(\mu z)[\chi\langle\mathfrak{f}, \mathfrak{g}\rangle(\mathfrak{x}, \mathfrak{y}, z)=0] .
\end{gathered}
$$

The case is similar, if the functional $\Phi\langle\mathfrak{f}\rangle_{x}$ is substituted for more func tion variables in the functional $\Omega$.

If $\Omega$ is obtained from $\Psi$ by means of the operation of identification of the function-variables, then our property is obviously satisfied by $\Omega$ because the inductive hypothesis has a stronger form. The operation of identification of the number-variables also presents no difficulties.

7. For each $\Phi \in \mathscr{K}$ there exists $\chi \in \mathscr{K}$ such that if

$$
F_{x}(y)=F(x, y) \quad \text { and } \quad f(x)=F(\text { Fi } x \text {, Se } x)
$$

Let us set

$$
\Phi\left\langle g, F_{x}\right\rangle\left(z_{1}, \ldots, z_{n}\right)=\chi\langle\mathrm{g}, f\rangle\left(x, z_{1}, \ldots, z_{n}\right) .
$$

hence

$$
\nabla\langle f\rangle(x, y)=\nabla\langle f\rangle_{x}(y)=f(\mathrm{~Pa}(x, y))
$$

(7) $\quad \nabla\langle f\rangle_{x}(y)=f(\mathrm{~Pa}(x, y))=F(\mathrm{Fi}(\mathrm{Pa}(x, y))$, Se $\mathrm{Pa}(x, y))=F(x, y)=F_{x}(y)$.

From property 6 it follows that there exists a functional $\chi \in \mathscr{K}$ such that, according to $(7)$,

$$
\begin{gathered}
\chi\langle\mathfrak{g}, f\rangle\left(x, z_{1}, \ldots, z_{n}\right)=\Phi\left\langle\mathfrak{g}, \nabla\langle f\rangle_{x}\right\rangle\left(z_{1}, \ldots, z_{n}\right) \\
=\Phi\left\langle\mathfrak{g}, F_{x}\right\rangle\left(z_{1}, \ldots, z_{n}\right) .
\end{gathered}
$$

8. The functional $\Phi$ is said to be computable in the Banach-Mazur ${ }^{\mathbf{8}}$ sense provided that if $G_{1}, \ldots, G_{k}$ are computable functions and $G_{i x}(y)=G_{i}(x, y)$, then the function

$$
f\left(x_{1}, \ldots, x_{k}, y_{1}, \ldots, y_{l}\right)=\Phi\left\langle G_{1} x_{1}, \ldots, G_{k} x_{k}\right\rangle\left(y_{1}, \ldots, y_{l}\right)
$$

is computable. The functionals of the class $\mathscr{K}$ are computable in the sense of Banach-Mazur.

s) See Mazur [7]. 
From property 7 it follows that there exists $\chi \in \mathscr{K}$ such that putting

we obtain

$$
\left.\left.g_{i}^{\prime} \cdot x\right)=G_{i}^{\prime} \text { Fi } x \text {, Se } x\right)
$$

$$
\Phi\left\langle G_{1} x_{1}, \ldots, G_{k} x_{k}\right\rangle\left(y_{1}, \ldots, y_{l}\right)=\chi\left\langle g_{1}, \ldots, g_{k}\right\rangle\left(x_{1}, \ldots, x_{k}, y_{1}, \ldots, y_{l}\right) .
$$

Hence the function $f$ is computable in $g_{1}, \ldots, g_{k}$ (Property 6). If $G_{1}, \ldots, G_{k} \in$ Com then $g_{1}, \ldots, g_{k} \in$ Com and $f \in$ Tom.

9. For each functional $\Phi \in \mathscr{K}$ there cxists another functional $\Omega_{\Phi} \in \mathcal{K}$ such that for any $f_{1}, \ldots, f_{n}, g_{1}, \ldots, g_{n}, x$

$$
\begin{gathered}
\text { if } g_{i}(u)=f_{i}(u) \text { for } u \leqslant \Omega_{\Phi}\left\langle f_{1}, \ldots, f_{n}\right\rangle(\mathfrak{x}), \\
\text { then } \Phi\left\langle f_{1}, \ldots, f_{n}\right\rangle(x)=\Phi\left\langle g_{1}, \ldots, \ell_{n}\right\rangle(x) .
\end{gathered}
$$

Proof by induction. If $\Phi$ is the initial identity functional $C$, then we have

$$
\text { if } \cdot g(u)=f(u) \quad \text { for } \quad u \leqslant x, \text { then } \quad U_{1}\langle f\rangle(x)=f(x)=g(x)=U_{1}\langle g\rangle(x),
$$

hence $\Omega_{U}=x$. For the constant initial functionals it is obvious that $\Omega_{-}=\Omega_{T}=\Omega_{S}=0$.

If $\Phi$ is obtained by means of the operation of substitution from the functionals $\Phi^{\prime}, \Phi^{\prime \prime} \in \mathscr{K}$, then the inductive hypothesis implies that

(9) $g_{i}(u)=f_{i}(u)$ for $u \leqslant \Omega_{\Phi^{\prime}}\left\langle f_{1}, \ldots, f_{n}\right\rangle\left\langle x_{1}, \ldots, x_{k-1}, \Phi^{\prime \prime}\left\langle f_{n+1}, \ldots, f_{t}\right\rangle\left(y_{1}, \ldots, y_{l}\right\rangle\right)$,

$$
\begin{aligned}
\text { then } \quad \Phi^{\prime}\left\langle f_{1}, \ldots, f_{n}\right\rangle\left\langle x_{1}, \ldots, x_{k-1}, \Phi^{\prime \prime}\left\langle f_{n+1}, \ldots, f_{t}\right\rangle\left(y_{1}, \ldots, y_{l}\right)\right) \\
=\Phi^{\prime}\left\langle g_{1}, \ldots, g_{n}\right\rangle\left\langle x_{1}, \ldots, x_{k-1}, \Phi^{\prime \prime}\left\langle f_{n+1}, \ldots, f_{t}\right\rangle\left(y_{1}, \ldots, y_{l}\right)\right) ;
\end{aligned}
$$

on the other hand, we have

$$
g_{i}(u)=f_{i}(u) \quad \text { for } \quad u \leqslant \Omega_{\Phi^{\prime \prime}}\left\langle f_{n+1}, \ldots, f_{t}\right\rangle\left(y_{1}, \ldots, y_{l}\right),
$$$$
\text { then } \Phi^{\prime \prime}\left\langle f_{n+1}, \ldots, f_{t}\right\rangle\left(y_{1}, \ldots, y_{l}\right)=\Phi^{\prime \prime}\left\langle g_{n+1}, \ldots, g_{t}\right\rangle\left(y_{1}, \ldots, y_{l}\right) \text {. }
$$

Thus, if we set

$$
\begin{gathered}
\Omega_{\Phi}\left\langle f_{1}, \ldots, f_{t}\right\rangle\left(x_{1}, \ldots, x_{k-1}, y_{1}, \ldots, y_{l}\right) \\
=\max \left[\Omega_{\Phi^{\prime}}\left\langle f_{1}, \ldots, f_{n}\right\rangle\left(x_{1}, \ldots, x_{k-1}, \Phi^{\prime \prime}\left\langle f_{n+1}, \ldots, f_{t}\right\rangle\left(y_{1}, \ldots, y_{l}\right)\right),\right. \\
\left.\Omega_{\Phi^{\prime \prime}}\left\langle f_{n+1}, \ldots, f_{t}\right\rangle\left(y_{1}, \ldots, y_{l}\right)\right]
\end{gathered}
$$

then from (9) and (10) it follows that

$$
\begin{gathered}
\text { if } g_{i}(u)=f_{i}(u) \text { for } u \leqslant \Omega_{\Phi}, \\
\text { then } \Phi^{\prime}\left\langle f_{1}, \ldots, f_{n}\right\rangle\left\langle x_{1}, \ldots, x_{k-1}, \Phi^{\prime \prime}\left\langle f_{n+1}, \ldots, f_{t}\right\rangle\left(y_{1}, \ldots, y_{l}\right)\right) \\
=\Phi^{\prime}\left\langle g_{1}, \ldots, g_{n}\right\rangle\left\langle x_{1}, \ldots, x_{k-1}, \Phi^{\prime \prime}\left\langle g_{n+1}, \ldots, g_{t}\right\rangle\left(y_{1}, \ldots, y_{l}\right)\right) .
\end{gathered}
$$

If $\Phi^{\prime}$ is obtained from $\Phi \in \mathscr{K}$ by means of the operation of identification of the variables, then $\Omega_{\Phi^{\prime}}$ is obviously obtained from $\Omega_{\Phi}$ by means of the same operation.

If $\Phi^{\prime}$ is obtained from $\Phi \in \mathscr{K}$ by means of the operation of effective minimum

$$
\begin{array}{rr}
\text { (11) } & \Phi^{\prime}\left\langle f_{1}, \ldots, f_{n}\right\rangle\left(x_{1}, \ldots, x_{k-1}\right)=(\mu y)\left[\Phi\left\langle f_{1}, \ldots, f_{n}\right\rangle\left(x_{1}, \ldots, x_{k-1}, y\right)=0\right] \\
\text { (12) } & \prod_{x_{1}, \ldots, x_{k-1} \in \mathcal{V}} \sum_{y \in \mathcal{Y N}} \Phi\left\langle f_{1}, \ldots, f_{n}\right\rangle\left(x_{1}, \ldots, x_{k-1}, y\right)=0 \\
\text { then } & \\
\text { (13) } & \Omega_{\Phi^{\prime}}\left\langle f_{1}, \ldots, f_{n}\right\rangle\left(x_{1}, \ldots, x_{k-1}\right)=\operatorname{Max} \Omega_{\Phi}\left\langle f_{1}, \ldots, f_{n}\right\rangle\left(x_{1}, \ldots, x_{k-1}, s\right) \\
& \text { for } s \leqslant \Phi^{\prime}\left\langle f_{1}, \ldots, f_{n}\right\rangle\left(x_{1}, \ldots, x_{k-1}\right) .
\end{array}
$$

Indeed if

$$
g_{i}(u)=f_{i}(u) \quad \text { for } \quad u \leqslant \Omega_{\Phi^{\prime}}\left\langle f_{1}, \ldots, f_{n}\right\rangle\left(x_{1}, \ldots, x_{k-1}\right),
$$

then from (13) by the influctive hypothesis it follows that

$$
\text { if } y \leqslant \Phi^{\prime}\left\langle f_{1}, \ldots, f_{n}\right\rangle\left(x_{1}, \ldots, x_{k-1}\right) \text {, }
$$

then $\Phi\left\langle f_{1}, \ldots, f_{n}\right\rangle\left(x_{1}, \ldots, x_{k-1}, y\right)=\Phi\left\langle g_{1}, \ldots, g_{n}\right\rangle\left(x_{1}, \ldots, x_{k-1}, y\right)$.

Formulae (11), (12), (15) imply that

(16) $\Phi\left\langle g_{1}, \ldots, g_{n}\right\rangle\left(x_{1}, \ldots, x_{k-1}, y\right) \neq 0 \quad$ for $\quad y<\Phi^{\prime}\left\langle f_{1}, \ldots, f_{n}\right\rangle\left(x_{1}, \ldots, x_{k-1}\right)$ and

(17) $\Phi\left\langle g_{1}, \ldots, g_{n}\right\rangle\left\langle x_{1}, \ldots, x_{k-1}, \Phi^{\prime}\left\langle f_{1}, \ldots, f_{n}\right\rangle\left(x_{1}, \ldots, x_{k-1}\right)\right\rangle=0$,

but (16) and (17) mean that

$$
\Phi^{\prime}\left\langle f_{1}, \ldots, f_{n}\right\rangle\left(x_{1}, \ldots, x_{k-1}\right)
$$$$
=(\mu y)\left[\Phi\left\langle g_{1}, \ldots, g_{n}\right\rangle\left(x_{1}, \ldots, x_{k-1}, y\right)=0\right]=\Phi^{\prime}\left\langle g_{1}, \ldots, g_{n}\right\rangle\left(x_{1}, \ldots, x_{k-1}\right),
$$

which completes the proof.

Let us set

$$
\begin{gathered}
\Gamma\langle f\rangle(n)(x)=\left\{\begin{array}{ccc}
f(x) & \text { for } & x \leqslant n, \\
0 & \text { for } & x>n,
\end{array}\right. \\
\Lambda(m)(x)=\Delta_{m}(x)=\exp \left(m+1, p_{x}\right), \\
\Lambda\langle f\rangle(n)=\left(\prod_{k \leqslant n} p_{k}^{f(k)}\right)-1 .
\end{gathered}
$$

10. Each functional $\Phi \in \mathscr{K}$ can be presented in the form

i.e.

$$
\Phi\left\langle f_{1}, \ldots, f_{n}\right\rangle(x)=\lim _{m \rightarrow \infty} g\left(\Lambda\left\langle f_{1}\right\rangle(m), \ldots, \Lambda\left\langle f_{n}\right\rangle(m), x\right),
$$$$
\Phi\left\langle f_{1}, \ldots, f_{n}\right\rangle(\mathfrak{x})=(\mu y)\left[\sum_{I \in \mathscr{N}} \prod_{m \geqslant l} g\left(\Lambda\left\langle f_{1}\right\rangle(m), \ldots, \Lambda\left\langle f_{n}\right\rangle(m), x\right)=y\right]
$$

where $g \in$ Com.

Fundamenta Mathematicae. T. XLIr. 
From property 9 and from the definition of $\Gamma$ it follows that for $m \geqslant \Omega_{\Phi}\left(f_{1}, \ldots, f_{n}\right\rangle(\mathfrak{x})$

$$
\Phi\left\langle f_{1}, \ldots, f_{n}\right\rangle(\mathfrak{x})=\Phi\left\langle\Gamma\left\langle f_{1}\right\rangle(m), \ldots, \Gamma\left\langle f_{n}\right\rangle(m)\right\rangle(\mathfrak{x}) .
$$

From property 8 it follows that there exists a function $g \in \mathbb{C o m}$ such that

$$
g\left(y_{1}, \ldots, y_{n}, \mathfrak{x}\right)=\Phi\left\langle\Delta y_{1}, \ldots, \Delta y_{n}\right\rangle(\mathfrak{x}) .
$$

On the other hand, the definitions of $\Gamma, \Delta, \Lambda$ imply that

$$
\Delta(\Lambda\langle f\rangle(m))(x)=\Gamma\langle f\rangle(m)(x)
$$

From (18), (19), and (20) we find that for $m \geqslant \Omega_{\Phi^{\prime}} f_{1}, \ldots, f_{n}>(\mathfrak{x})$

$$
g\left(\Lambda\left\langle f_{1}\right\rangle(m), \ldots, \Lambda\left\langle f_{n}\right\rangle(m), \mathfrak{x}\right)=\Phi\left\langle f_{1}, \ldots, f_{n}\right\rangle(\mathfrak{x}),
$$

which completes the proof.

11. There exists a functional $\Psi$ which can be presented in the form (A) and which is not computable in the Banach-Mazur sense. Hence $\Psi \dot{F}$.

We put

$$
g(0)=0, \quad g(n+1)=1, \quad \Psi\langle f\rangle=\lim _{m \rightarrow \infty} g(\Lambda\langle f\rangle(m)) .
$$

The functional $\Psi$ is well defined for any function:

$$
\text { if } \prod_{x \in \mathscr{N}} f(x)=0 \text { then } \prod_{m \in \mathfrak{N}} g(\Lambda\langle f\rangle(m))=0 \text { and } \Psi\langle f\rangle=0 \text {, }
$$

if $\sum_{x \in \mathfrak{F}} f(x) \neq 0$ then $\sum_{x \in \mathscr{S N}} \prod_{m \geqslant x} g(\Lambda\langle f\rangle(m))=1$ and $\Psi\langle f\rangle \neq 0$.

Hence it is true that

$$
\Psi\langle f\rangle=0 \equiv \prod_{x \in \mathscr{V}} f(x)=0 .
$$

Mazur [7] has pointed out that the functionals which satisfy the condition (21) are not computable in the Banach-Mazur sense. Thus from 8 it follows that $\Psi \notin \mathscr{K}$.

The functionals of Banach and Mazur possess property 10 for $f_{1}, \ldots, f_{n} \in$ (rom. It is a very interesting question whether any functional defined for all functions by means of the form (A) and computable in the sense of Banach and Mazur belongs to the class $\mathcal{K}$.

Let us put

$$
\begin{aligned}
& 3\langle f\rangle=\underset{g \in \mathcal{E}}{E}\left[\prod_{x \in \mathfrak{V}} g(x) \leqslant f(x)\right], \\
& v_{\Phi}\langle f\rangle\left(x_{1}, \ldots, x_{k}\right)=(\mu z)\left[\prod_{g_{1}, \ldots, g_{n}, h_{1}, \ldots, h_{k} \in 3\langle f\rangle} \text { if } g_{i}(u)=h_{i}(u)\right. \\
& \text { for } \left.u \leqslant z \text { then } \Phi\left\langle g_{1}, \ldots, g_{n}\right\rangle\left(x_{1}, \ldots, x_{k}\right)=\Phi\left\langle h_{1}, \ldots, h_{n}\right\rangle\left(x_{1}, \ldots, x_{k}\right)\right] \text {. }
\end{aligned}
$$

12. The class $\mathscr{K}$ is closed under the following scheme of functional induction: if $\chi, \Psi, x \in \mathscr{K}, J$ is a functional not necessarily computable and
(a) $\Phi\langle f\rangle_{0}(x)=\chi\langle f\rangle(x)$,
(b) $\Phi\langle f\rangle_{n+1}(x)=\Psi\left\langle\Phi\langle f\rangle_{n}\right\rangle\langle(x)$,
(c) $\left.\Phi\langle f\rangle_{n}^{\prime}, x\right) \leqslant J\langle f\rangle(x)$,
(d) $y_{\Psi}\langle J\langle f\rangle\rangle(x) \leqslant x\langle f\rangle(x)$ for any $f$ and $x$,

then $\Phi \in \mathscr{K}$.

Indeed, according to (d), we have

$$
\left.\left.\prod_{g \in 3\langle J\langle f\rangle} \Psi\langle g\rangle(x)=\Psi\langle\Gamma\langle g\rangle| x\langle f\rangle(x)\right)\right\rangle(x) ;
$$

hence in conformity with the definitions of $\Gamma, \Lambda, A$,

$$
\prod_{g \in 3\langle J\langle f\rangle\rangle} \Psi\langle g\rangle(x)=\Psi\langle\Delta(\Lambda\langle g\rangle\langle x\langle f\rangle(x)))\rangle(x) .
$$

Condition (c) implies that for any $f \in \mathfrak{F}, \Phi\langle f\rangle_{n} \in \mathfrak{Z}\langle J\langle f\rangle\rangle$. Hence from (23) and (b) it follows that

(24) $\prod_{f \in \Im} \prod_{x \in \mathscr{S}} \Phi\langle f\rangle_{n+1}(x)=\Psi\left\langle\Delta\left(\Lambda\left\langle\Phi\langle f\rangle_{n}\right\rangle\langle x\langle f\rangle(x))\right)\right\rangle(x)$.
Let us put

$$
\operatorname{Exp}(m, x)=\exp \left(m, p_{x}\right) \text {. }
$$

It is evident that the following condition is true:

(25) $\prod_{f \in \mathcal{F}} \prod_{n, x \in \mathfrak{N} N} \sum_{m \in \mathscr{N}}$,

A. $\prod_{t, s \leqslant m}\{0<t=\operatorname{Exp}(m, \mathrm{~Pa}(0, s)) \rightarrow t=\chi\langle f\rangle(s)+1\}$,

B. $\prod_{r, r, s \leqslant m}\{<t=\operatorname{Exp}(m, \mathrm{~Pa}(r+1, s))$

$$
\left.\rightarrow t=1+\Psi\left\langle\Delta\left(\left(\prod_{i \leqslant k\langle f\rangle(s)} L_{i}^{\operatorname{Exp}(m, \operatorname{Pa}(r, i))-1}\right)-1\right)\right\rangle(s)\right\},
$$

C. $\prod_{t, r, s \leqslant m} \prod_{i \leqslant \kappa\langle f\rangle(s)}\{0<t=\operatorname{Exp}(m, \mathrm{~Pa}(r+1, s))$

D. $\operatorname{Exp}(m, \operatorname{Pa}(n, x))>0$.

$$
\rightarrow \operatorname{Exp}(m, \operatorname{Pa}(r, i))>0\},
$$

Condition (25) can be considered as a condition of effectiveness, hence the following functional $\Psi^{\prime}$ is computable:

$$
\Psi^{\prime}\langle f\rangle(n, x)=(\mu m)[\text { A. B. C. D. }(m, n, x, f)] .
$$

We shall prove by induction that

(26) $\prod_{t, r, s \leqslant \Psi^{\prime}\langle f\rangle(n, x)} 0<t=\operatorname{Exp}\left(\Psi^{\prime}\langle f\rangle(n, x), \mathrm{Pa}_{1}(r, s)\right) \rightarrow t=\Phi\langle f\rangle_{r}(s)+1$ 
Condition (26) is obvious for $r=0$ and for any $s, t$ according to A and (a). Now let us suppose that condition (26) is satisfied for $r$ and for any $s$ and $t$.

From (26) and $\mathrm{C}$ it follows that

$$
\prod_{i \leqslant \kappa\langle f\rangle(s)} p_{i}^{\operatorname{Exp}\left(\Psi^{\prime}\langle f\rangle(n, x), \operatorname{Pa}(r, i)\right)-1}=\Lambda\left\langle\Phi\langle f\rangle_{r}\right\rangle(x\langle f\rangle(s))-1 ;
$$

hence from (27), B and (24) it follows that

$$
\begin{gathered}
\text { if } 0<t \quad \text { and } t=\operatorname{Exp}\left(\Psi^{\prime}\langle f\rangle(n, x), \mathrm{Pa}^{\prime}(r+1, s)\right), \\
\text { then } t=1+\Psi\left\langle\Delta\left(\Lambda\left\langle\Phi\langle f\rangle_{r}\right\rangle\right)(x\langle f\rangle(s))\right\rangle(s)^{\prime} \text { and } t=\Phi\langle f\rangle_{r+1}(s),
\end{gathered}
$$

which completes the inductive proof.

Thus, arcording to condition D, the functional $\Phi$ can be defined as follows:

$$
\Phi\langle f\rangle_{n}(x)=\operatorname{Exp}\left(\Psi^{\prime}\langle f\rangle(n, x), \operatorname{Pa}(n, x)\right)-1 .
$$

The problem whether the general scheme of functional induction (i,e. the conditions (a) and (b)) leads out of the class $\mathscr{K}$ remains open.

\section{3. Uniformity Theorem}

For the applications to the analysis the following Uniformity Theorem is very important:

UNIFORMTIY THEOREM. For each functional $\Phi \in \mathscr{K}$ there exists a functional $\omega_{\Phi} \in \mathscr{K}$ such that for any $x \in \mathscr{N}$ and $f \in \mathfrak{F}$ the following condition is satisfied:

$$
\begin{aligned}
& \prod_{g_{1}, \ldots, g_{n}, h_{1}, \ldots, h_{n} \in B\langle f\rangle} \text { if } g_{i}(u)=h_{i}(u) \text { for } u \leqslant \omega_{\Phi}\langle f\rangle(x) \\
& \text { then } \Phi\left\langle g_{1}, \ldots, g_{n}\right\rangle(x)=\Phi\left\langle h_{1}, \ldots, h_{n}\right\rangle(x)
\end{aligned}
$$

where $3\langle f\rangle$ is the set of all functions $g \in \mathfrak{F}$ dominated by $f$ :

$$
g \in \mathcal{Z}\langle f\rangle \equiv \prod_{x \in \operatorname{Sr}} g(x) \leqslant f(x)
$$

Proof. To symplify the proof let us suppose that $n=1, i$. e. that $\Phi$ is a functional of one function-argument. The proof is topological. We define a mapping $a(g)$ of the set $3\langle f\rangle$ of functions upon the closed segment $[0,1]$ :

$$
\alpha(g)=\sum_{x=0}^{\infty} \frac{g(x)}{\prod_{i \leqslant x}(f(i)+1)}=\frac{g(0)}{f(0)+1}+\frac{g(1)}{(f(0)+1)(f(1)+1)}+\ldots
$$

Let us put

$$
T_{f}=E_{a}\left[a=1 \quad \text { or } \quad \sum g \in 3\langle f\rangle=\alpha(g)\right]
$$

From these definitions it follows that there are two eases:

( $\alpha$ ) The function $f$ assumes the values $f\left(n_{i}\right)>0$ only for a finite number of arguments $n_{0}, \ldots, n_{k} \in \mathscr{N}$. Then the set $T_{f}$ is finite.

$(\beta)$ The function $f$ assumes the values $f(n)>0$ for infinitely many $n \in \mathscr{N}$. Then $T_{f}$ is the closed segment $T_{f}=[0,1]$.

In any case $T_{f}$ contains two numbers, 0 and 1 . Obviously $T_{f} \subset[0,1]$.

There are two kinds of numbers of the set $T_{f}$ : rational numbers in $f$ and irrational numbers in $f$.

The number $a$ is irrational in $f$ provided that there exists a function $g_{a} \in 3\langle f\rangle$ such that $a=\alpha\left(g_{a}\right)$ and $0<g_{a}(n)<f(n)$ for infinitely many $n \in \mathscr{N}$. In the contrary case the number $a \in T_{f}$ is said to be rational in $f$. It is evident that the set $T_{f}$ is finite if and only if all elements of the set $T_{f}$ are rational in $f$. If $a$ is irrationsl in $f$, then $f(n)>0$ for infinitely many $n \in \mathscr{N}$, hence $T_{f}=[0,1]$. If $T_{f}=[0,1]$, then the set of rationals in $f$ is denumerable and if $1 \neq a \neq 0$ is a rational number in $f$, then there exist two functions $s_{a}^{\prime},,_{a}^{\prime \prime} \in \mathcal{Z}\langle f\rangle$ and an integer $k_{0} \in \mathscr{N}$ such that $a=a\left(g_{a}^{\prime}\right)=$ $=\alpha\left(g_{a}^{\prime \prime}\right)$ and

$$
\begin{array}{r}
g_{a}^{\prime}(x)=\left\{\begin{array}{ccc}
g_{a}^{\prime \prime}(x) & \text { for } x<k_{0}, \\
g_{a}^{\prime \prime}(x)-1 & \text { for } x=k_{0}, \\
f(x) & \text { for } x>k_{0},
\end{array}\right. \\
g_{a}^{\prime \prime}(x)=\left\{\begin{array}{ccc}
g_{a}^{\prime}(x) & \text { for } x<k_{0}, \\
g_{a}^{\prime}(x)+1 & \text { for } x=k_{0}, \\
0 & \text { for } x>k_{0} .
\end{array}\right.
\end{array}
$$

If $a$ is irrational in $f$, then there exists exactly one $g_{a} \in \mathcal{Z}\langle f\rangle$ such that $a=\alpha\left(g_{a}\right)$.

Now we shall show that there are two functionals $\Psi^{\prime}, \Psi^{\prime \prime} \in \mathscr{K}$ such that if $T_{f}=[0,1]$ then for each $1 \neq a \neq 0$ if $a$ is rational in $f$ then there exists such $n \in \mathscr{N}$ that, for $\Psi\langle f\rangle_{n}(x)=\Psi\langle f\rangle(n, x)$,

$$
a=\alpha\left(\Psi^{\prime}\langle f\rangle_{n}\right)=\alpha\left(\Psi^{\prime \prime}\langle f\rangle_{n}\right) \quad \text { and } \quad g_{a}^{\prime}=\Psi^{\prime}\left\langle f_{\rangle_{n}}, \quad g_{a}^{n}=\Psi^{\prime \prime}\langle f\rangle_{n} .\right.
$$

The definitions of the functionals $\Psi^{\prime}$ and $\Psi^{\prime \prime}$ are recursive:

$$
\Phi_{1} f>(0, k, x)=0
$$$$
\dot{\Phi}_{1} f,(n+1, k, 0)=\left\{\begin{array}{l}
0 \text { if } \Phi_{1}\langle f\rangle(n, k, 0)=f(0), \\
\Phi_{1}\langle f\rangle(n, k, 0)+1 \text { in the contrary case. }
\end{array}\right.
$$

$$
\Phi_{1}\langle f\rangle(n+1, k, x+1)=\left\{\begin{array}{r}
\Phi_{1}\langle f\rangle(n, k, x+1) \text { if } x+1 \leqslant k \\
\text { and } \sum_{z \leqslant x} \Phi_{1}\langle f\rangle(n, k, z)<f(z), \\
\Phi_{1}\langle f\rangle(n, k, x+1)+1 \text { if } x+1 \leqslant k, \\
\Phi_{1}\langle f\rangle(n, k, x+1)<f(x+1) \\
\text { and } \prod_{z \leqslant x} \Phi_{1}\langle f\rangle(n, k, z)=f(z), \\
0 \text { if } x+1>k \text { or } \prod_{z \leqslant x+1} \Phi_{1}\langle f\rangle(n, k, z)=f(z) .
\end{array}\right.
$$




$$
\Phi_{2}\langle f\rangle(n, k, x)=\left\{\begin{array}{r}
\Phi_{1}\langle f\rangle(n, k, x) \text { if } x<k \text { and } \sum_{x<z \leqslant k} \Phi_{1}\langle f\rangle(n, k, z) \cdots 0, \\
\Phi_{1}\langle f\rangle(n, k, x)-1 \text { if } x \leqslant k \text { and } \Phi_{1}\langle f\rangle(n, k, x)>0 \\
f(x) \text { if } x>k \text { or } x<k \text { and } \prod_{x<z \leqslant k} \Phi_{1}\langle f\rangle(n, k, z)=0, \\
\quad \prod_{x \leqslant z \leqslant k} \Phi_{1}\langle f\rangle(n, k, z)=0 .
\end{array}\right.
$$

$$
\Phi_{3}\langle f\rangle(x)=\sum_{s \leqslant x} \prod_{i \leqslant s}(f(i)+1) \text {. }
$$

$$
\Phi_{4}\langle f\rangle(x)=(\mu z)\left[x<\Phi_{3}\langle f\rangle(z+1)\right] .
$$

$$
\Psi^{\prime}\langle f\rangle(n, x)=\Phi_{2}\langle f\rangle\left(n-\Phi_{3}\langle f\rangle\left(\Phi_{4}\langle f\rangle(n)\right), \Phi_{\mathbf{4}}\langle f\rangle(n), n\right),
$$$$
\Psi^{\prime \prime}\langle f\rangle(n, x)=\Phi_{1}\langle f\rangle\left(n-\Phi_{3}\langle f\rangle\left(\Phi_{4}\langle f\rangle(n)\right), \Phi_{4}\langle f\rangle(n), x\right) .
$$

It is easy to show that the class $\mathscr{K}$ is closed under the operation of double induction used in the definition of the functional $\left.\Phi_{1}{ }^{9}\right)$. Hence $\Psi^{\prime}, \Psi^{\prime \prime} \in \mathcal{K}$.

From (5) it follows that each number of the form $n_{0}^{\prime}(f(0)+1)$ for $0 \leqslant n_{\theta} \leqslant f(0)$ can be presented in the form $a\left(\Phi_{1}\langle f\rangle_{n_{0}, 0}\right)$ where $\Phi_{1}\langle f\rangle_{n, k}(x)=$ $=\Phi_{1}\langle f\rangle(n, k, x)$. Similarly each number of the form

$$
\frac{n_{0}}{f(0)+1}+\frac{n_{1}}{(f(0)+1)(f(1)+1)}<1
$$

can be presented as $\alpha\left(\Phi_{1}\langle f\rangle_{m, 1}\right)$ for $0 \leqslant m \leqslant(f(0)+1)+(f(0)+1)(f(1)+1)$.

In general let the numbers of the form

$$
a=\sum_{j \leqslant k} \frac{n_{j}}{\prod_{i \leqslant j}(f(i)+1)} \quad \text { and } \quad a<1
$$

be called the numbers of k-order. Each number of $k$-order can be presented in the form $\alpha\left(\Phi_{1}\left\langle f_{m_{m}, k}\right)\right.$ for some $m \in \mathscr{N}$ such that $0 \leqslant m \leqslant \Phi_{3}\langle f\rangle(k)$ Hence according to (7)-(9) this number can be presented as $\alpha\left(\Psi^{\prime \prime \prime}\langle f\rangle_{n}\right)$ where $n=m+\Phi_{3}\langle f\rangle(k)$ and $\Psi^{\prime \prime}\langle f\rangle_{n}(x)=\Psi^{\prime \prime}\langle f\rangle(n, x)$, because from $(7)-(9)$ it follows that

hence

$$
\begin{gathered}
\Phi_{4}\langle f\rangle\left\langle m+\Phi_{3}\langle f\rangle(k)\right)=k \quad \text { and } \quad n-\Phi_{3}\langle f\rangle(k)=m, \\
\Psi^{\prime \prime}\langle f\rangle\left(m+\Phi_{3}\langle f\rangle(k), x\right)=\Phi_{1}\langle f\rangle(m, k, x) .
\end{gathered}
$$

Each number rational in $f$ can also be presented as $\alpha\left(\Psi^{\prime \prime}\langle f\rangle_{n}\right)$ for some $n \in \mathfrak{N}$.

9) The functional $\Phi_{1}$ is defined by means of a double recursion without entanglements. This scheme can be reduced to the scheme of simple recursion. Cf. Peter [10], [11].
If the set $T_{f}$ is finite then for a number $1 \neq a \in T_{f}$ there exists such $n \epsilon \mathscr{N}$ that $a=\alpha\left(\Psi^{\prime \prime}\langle f\rangle_{n}\right)$ and $\Psi^{\prime \prime}\langle f\rangle_{n}=g_{a}^{\prime \prime}$ but $\alpha\left(\Psi^{\prime \prime}\langle f\rangle_{n}\right) \neq \alpha\left(\Psi^{\prime}\langle f\rangle_{n}\right)$ and $\Psi^{\prime}\langle j\rangle_{n} \neq g_{a}^{\prime}$. Namely, the number $\alpha\left(\Psi^{\prime}\langle f\rangle_{n}\right)$ is the immediate predecessor of the number $\alpha\left(\Psi^{\prime \prime}\langle f\rangle_{n}\right)$. Indeed, according to $(\alpha)$, if $T_{f}$ is finite then there existis such a $k_{1}$ that

$$
k_{1}=(\mu v)\left[\prod_{x>z} f(x)=0\right] .
$$

If $k_{1}=0$, then the set $T_{f}$ contains only one function $f$ and for all $x \in \mathscr{N}$, $f(x)=0$, hence the set $T_{f}$ contains only two numbers: 0 and 1 . If $k_{1}>0$ then the set $T_{f}$ contains more than two points. Namely, it is true that

$$
\overline{\bar{T}_{f}}=1+\prod_{i \leqslant k_{1}}(f(i)+1) \text {. }
$$

The numbers of the set $T_{f}$ can be presented in the form

$$
a_{t}=\frac{t}{\prod_{i \leqslant k_{1}}(f(i)+1)} \quad \text { where } \quad 0 \leqslant t \leqslant \prod_{i \leqslant k_{1}}(f(i)+1) .
$$

From the definitions of the functionals $\Psi^{\prime}$ and $\Psi^{\prime \prime}$ it follows that

and

$$
\text { if } a_{t} \neq 0 \text { and } a_{t}=\alpha\left(\Psi^{\prime \prime}\langle f\rangle_{n}\right) \text { then } a_{t-1}=\alpha\left(\Psi^{\prime}\langle f\rangle_{n}\right) \text {, }
$$

$$
\text { if } \quad a_{t} \neq 0 \text { and } a_{t}=\alpha\left(\Psi^{\prime \prime}\langle f\rangle_{n}\right) \text { then } g_{a_{t-1}}^{n}=\Psi^{\prime}\langle f\rangle_{n} .
$$

If $T_{f}=[0,1]$, then it is evident that, according to $(6)$, if $a$ can be presented as $\alpha\left(\Phi_{1}\langle f\rangle_{m, k}\right)$ then $a$ can also be presented as $a\left(\Phi_{2}\langle f\rangle_{m, k}\right)$ where $\Phi_{2}\langle f\rangle_{m, k}(x)=\Phi_{2}\langle f\rangle(m, k, x)$, and hence $g_{a}^{\prime}=\Phi_{2}\langle f\rangle_{m, k}=\Psi^{\prime}\langle f\rangle_{m+\Phi_{3}}\langle f\rangle(k)$.

It is obvious that in any case $a\left(\Psi^{\prime \prime}\langle f\rangle_{0}\right)=0$.

Hence we can define a sequence $r_{n}$ of all rationals in $f$ :

$$
r_{0}=1, \quad r_{n+1}=\alpha\left(\Psi^{\prime \prime}\langle f\rangle_{n}\right) .
$$

We have $r_{0}=1, r_{1}=0$. Each rational number in $f$ appears in this sequence infinitely many times.

Now for each number $r_{n}$ rational in $f$ we find an open neighbourhood $O_{r_{n}}$ and an integer $s\left(r_{n}\right)$ such that

(15) $\prod_{g, h \in 3\langle f\rangle}$ if $a(g), \alpha(h) \in O_{r_{n}}$ and $g(u)=h(u)$ for $u \leqslant s\left(r_{n}\right)$,

We set

then $\Phi\langle g\rangle(\mathfrak{x})=\Phi\langle h\rangle(\mathfrak{x})$

(16) $\quad \Omega_{\Phi}^{*}\langle f, j\rangle(x)=(\mu s)\left[\prod_{g \in 3\langle f\rangle}\right.$ if $g(u)=j(u)$ for $u \leqslant s$, then $\Phi\langle j\rangle(\mathfrak{x})=\Phi\langle g\rangle(\mathfrak{x})$ 


$$
\begin{aligned}
s^{\prime}\left(r_{n}\right) & =\Omega_{\Phi}^{*}\left\langle f, \Psi^{\prime}\langle f\rangle_{n-1}\right\rangle(\mathfrak{x}), \\
s^{\prime \prime}\left(r_{n}\right) & =\Omega_{\Phi}^{*}\left\langle f, \Psi^{\prime \prime}\left\langle f_{{ }_{n-1}}\right\rangle(\mathfrak{x}),\right.
\end{aligned}
$$$$
s\left(r_{n}\right)=\max \left[s^{\prime}\left(r_{n}\right), s^{\prime \prime}\left(r_{n}\right)\right] \text {. }
$$

$$
\left.O_{1}=O_{r_{0}}=E_{a}\left[1 \geqslant a>1-\frac{1}{\prod_{i \leqslant s_{0}}(f(i)+1)}\right] \quad \text { where } s_{0}=\Omega_{\Phi}^{*} f, f\right\rangle(x),
$$

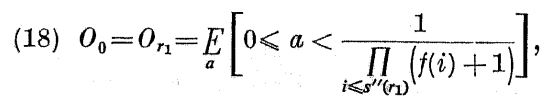

$$
O_{r_{n}}=\underset{a}{E}\left[r_{n}-\frac{1}{\prod_{i \leqslant s^{\prime}\left(r_{n}\right)}(f(i)+1)}<a<r_{n}+\frac{1}{\prod_{i \leqslant s^{\prime \prime}\left(r_{n}\right)}(f(i)+1)}\right] \text { for } 1 \neq r_{n} \neq 0 \text {. }
$$

It is evident that the set $O_{r_{n}}$ is open in the segment $[0,1]$ and $r_{n} \in O_{r_{n}}$. Now let us suppose that $h \in 3\langle f\rangle$ and

$$
\alpha(h) \in O_{r_{n}} ;
$$

from (5)-(9) and (16)-(18) it follows that for $r_{n} \neq 1$

$(20)$ if $a(h) \geqslant r_{n}$ then $h(u)=\Psi^{\prime \prime}\langle f\rangle_{n-1}(u)$ for $u \leqslant s^{\prime \prime}\left(r_{n}\right)$,

(21) if $a(h)<r_{n}$ then $h(u)=\Psi^{\prime}\langle f\rangle_{n-1}(u)$ for $u \leqslant s^{\prime}\left(r_{n}\right)$.

Indeed, $r_{n}=\alpha\left(\Psi^{\prime \prime}\langle f\rangle_{n-1}\right)$ according to (14), and $\Psi^{\prime \prime}\langle f\rangle_{n-1}=g_{r_{n}}^{\prime \prime}$ according to the definition of $\Psi^{\prime \prime}$; hence if $(19)$ and $\alpha(h) \geqslant r_{n}$ then

$$
r_{n} \leqslant \alpha(h)<r_{n}+\frac{1}{\prod_{i \leqslant s^{\prime \prime}\left(r_{n}\right)}(f(i)+1)} \quad \text { and } \quad h(u)=g_{r_{n}}(u)=\Psi^{\prime \prime}\langle f\rangle_{n-1}(u)
$$

$$
\text { for } u \leqslant s^{\prime \prime}\left(r_{n}\right) \text {. }
$$

In order to prove (21) we shall distinguish two cases:

(x) The set $T_{f}$ is finite. Suppose that (10)-(13) and that $r_{n}=a_{t}$ and $t \neq 0$. (For $r_{n}=0,(21)$ is vacuuosly satisfied.) Let us consider two cases: $s^{\prime}\left(r_{n}\right) \geqslant k_{1}$ and $s^{\prime}\left(r_{n}\right)<k_{1}$. If $s^{\prime}\left(r_{n}\right) \geqslant k_{1}$ then $\prod_{i \leqslant s^{\prime}\left(r_{n}\right)}(f(i)+1)=\prod_{i \leqslant k_{1}}(f(i)+1)$; hence, according to (11) and (12), it follows that there are no numbers $a \in T_{f}$ such that $a \in O_{r_{n}}$ and $a<r_{n}$. Then (21) is vacuuosly satisfied. If $s^{\prime}\left(r_{n}\right)$ $<k_{1}$ then we define the function $f^{*}: f^{*}(x)=f(x)$ for $x \leqslant k_{1}$ and $f^{*}\left(x+k_{1}+1\right)$ $=0$; hence thereare two functions $g_{r_{n}}^{\prime}, g_{r_{n}}^{\prime \prime}$ which satisfy (3) and (4) with respectto $f^{*}$, and $r_{n}=a\left(g_{r_{n}}^{\prime}\right)=\alpha\left(g_{r_{n}}^{\prime \prime}\right)$. The numbers $a(h) \in T_{f}$ such that

$$
r_{n}>\alpha(h)>r_{n}-\frac{1}{\prod_{i \leqslant s^{\prime}\left(r_{n}\right)}(f(i)+1)}
$$

have the following property:

$$
h(u)=g_{r_{n}}^{\prime}(u) \text { for } u \leqslant s^{\prime}\left(r_{n}\right) .
$$

On the other hand, (12) and (13) imply that

$$
\varrho_{r_{n}}^{\prime}(u)=\Psi^{\prime}\left\langle f_{n_{n-1}}(u) \text { for } u \leqslant k_{1} .\right.
$$

Now if $s^{\prime}\left(r_{n}\right)<k_{1}$ then from (22) and (23) it follows that

$$
h(u)=\Psi^{\prime}\langle f\rangle_{n-1}(u) \text { for } u \leqslant s^{\prime}\left(r_{n}\right) .
$$

(ß) $T_{f}^{\prime}=[0,1]$. Then $\Psi^{\prime}\langle f\rangle_{n-1}=\ell^{\prime} r_{n}$ for $n>1$. Hence it is evident that if $\alpha(h) \epsilon O_{r_{n}}$ and $a(h)<r_{n}, i$. $e$. if

$$
r_{n}>\alpha(h)>r_{n}-\frac{1}{\prod_{i \leqslant s^{\prime}\left(r_{n}\right)}(f(i)+1)},
$$

then $h(u)=g_{r_{n}}^{\prime}(u)=\Psi^{\prime}\langle f\rangle_{n-1}(u)$ for $u \leqslant s^{\prime}\left(r_{n}\right)$.

Now in order to prove (15) we set

$$
k_{2}=(\mu x)\left[\Psi^{\prime \prime}\langle f\rangle_{n-1}(u)=\Psi^{\prime}\langle f\rangle_{n-1}(u) \quad \text { for } . u<x\right]
$$

and we consider two cases: $k_{2} \geqslant s\left(r_{n}\right)$ or $k_{2}<s\left(r_{n}\right)$.

If $k_{2} \geqslant s\left(r_{n}\right)$ then $\Psi^{\prime \prime}\left\langle f_{\rangle_{n-1}}(u)=\Psi^{\prime}\langle f\rangle_{n-1}(u)\right.$ for $u \leqslant s\left(r_{n}\right)$; hence from (16) and (17) it follows that $s^{\prime}\left(r_{n}\right)=s^{\prime \prime}\left(r_{n}\right)=s^{\prime}\left(r_{n}\right)$. Thus if $a(h), a(g) \in O_{r_{n}}$, then, according to $(18), h(u)=\Psi^{\prime \prime}\langle f\rangle_{n-1}(u)=g(u)$ for $u \leqslant s\left(r_{n}\right)$ and by $(16)$

$$
\Phi\langle h\rangle(\mathfrak{x})=\Phi\left\langle\Psi^{\prime \prime}\langle f\rangle_{n-1}\right\rangle(\mathfrak{x})=\Phi\langle g\rangle(x) .
$$

If $k_{2}<s\left(r_{n}\right)$ and $a(h), \alpha(g) \in O_{r_{n}}$ and $h(u)=g(u)$ for $u \leqslant s\left(r_{n}\right)$, then, according to (20) and (21), it is possible only if $G(h) \geqslant r_{n}$ and $a(g) \geqslant r_{n}$ or $\alpha(h)<r_{n}$ and $\alpha(g)<r_{n}$. In the first case (20) implies that $h(u)=$ $=\Psi^{\prime \prime}\left\langle f_{n-1}^{\prime}(u)=g(u)\right.$ for $u \leqslant s^{\prime \prime}\left(r_{n}^{\prime}\right)$ and by (16)

$$
\Phi\langle h\rangle(x)=\Phi\left\langle\Psi^{\prime \prime}\left\langle f_{i n-1}\right\rangle(x)=\Phi\langle g\rangle(x) .\right.
$$

In the second case (21) implies that $h(u)=\Psi^{\prime}\left\langle f_{n-1}(u)=g(u)\right.$ for $u<s^{\prime}\left(r_{n}\right)$ and by $(16)$

$$
\Phi\langle h\rangle(\mathfrak{x})=\Phi\left\langle\Psi^{\prime}\langle f\rangle_{n-1}\right\rangle(\mathfrak{x})=\Phi\langle g\rangle(\mathfrak{x}) .
$$

Condition (15) is also proved for $1 \neq r_{n} \neq 0$.

For $r_{n}=1$ it is evident that if $a(h), \alpha(g) \in O_{r_{0}}$ then $h(u)=f(u)=g(u)$ for $u \leqslant s_{0}$; hence by $(16)$

$$
\Phi\langle h\rangle(\mathfrak{x})=\Phi\langle f\rangle(\mathfrak{x})=\Phi\langle\mathfrak{g}\rangle(\mathfrak{x}) .
$$

For $r_{n}=0$ it is also evident that if $\alpha(h), \alpha(g) \in O_{r_{1}}$, then, according to $(17),(5)$ and $(9), h(u)=\Psi^{\prime \prime}\langle f\rangle_{0}(u)=g(u)=0$ for $u \leqslant s^{\prime \prime}\left(r_{1}\right)$. Then by (16)

$$
\Phi\langle h\rangle(\mathfrak{x})=\Phi\left\langle\Psi^{\prime \prime}\langle f\rangle_{0}\right\rangle(\mathfrak{x})=\Phi\langle g\rangle(\mathfrak{x}) .
$$


Now we shall prove that the sequence of open sets $O_{r_{n}}$ covers the segment $[0,1]$. Let us distinguish two cases, $(\alpha)$ and $(\beta)$.

$(\alpha)$ The set $T_{f}$ is finite. Then for a number $r_{n}=a_{t}$ it follows from (10) that if $s^{\prime \prime}\left(r_{n}\right) \geqslant k_{1}$, then $\prod_{i \leqslant s^{\prime}\left(r_{n}\right)}(f(i)+1)=\prod_{i \leqslant k_{1}}(f(i)+1)$; on the other hand, if $k_{1}>s^{\prime \prime}\left(r_{n}\right)$, then $\prod_{i \leqslant s^{\prime \prime}\left(r_{n}\right)}(f(i)+1)<\prod_{i \leqslant k_{1}}(f(i)+1)$. Similarly for $s^{\prime}\left(r_{n}\right)$. Hence for each $r_{n}$

$$
\prod_{i \leqslant s^{\prime \prime}\left(r_{n}\right)}(f(i)+1) \leqslant \prod_{i \leqslant k_{1}}(f(i)+1) \geqslant \prod_{i \leqslant s^{\prime}\left(r_{n}\right)}(f(i)+1) .
$$

Thus by (11) and (18) it follows that, for $1 \neq r_{n} \neq 0$,

and

$$
\underset{a}{E}\left[c_{t-1}<a<c_{t+1}\right] \subset O_{r_{n}}
$$

$$
\underset{a}{E}\left[0 \leqslant a_{1}<a_{1}\right] \subset O_{r_{1}}, \quad \underset{a}{E}\left[1 \geqslant a>1-a_{1}\right] \subset O_{r_{0}} .
$$

Also according to (11) the sets $O_{r_{n}}$ cover the segment $[0,1]$.

( $\beta) T_{f}=[0,1]$. Then it suffices to show that for each number $b$ irrational in $f$ there exists such an $n \in \mathscr{N}$ that $b \in O_{r_{n}}$. Indeed, let $b=\alpha(g)$ and $k_{3}=\Omega_{\Phi}^{*}\langle g\rangle(\mathfrak{x})$. It is evident that there exists a number $a=r_{n}$, irrational in $f$, such that according to (3)-(9)

$$
g_{a}^{\prime \prime}(u)=\Psi^{\prime \prime}\langle f\rangle_{n-1}(u)=\Gamma\langle g\rangle\left(k_{3}\right)(u) .
$$

Now we prove that $b \in O_{r_{n}}$. From (24) and (16) it follows that

$$
g_{a}^{\prime \prime}(u)=\Psi^{\prime \prime}\langle f\rangle_{n-1}(u)=g(u) \quad \text { for } \quad u \leqslant k_{3},
$$

(26) $\quad \prod_{h \in \mathcal{F}}$ if $h(u)=g(u)$ for $u \leqslant k_{3}$, then $\Phi\langle h\rangle(\mathfrak{x})=\Phi\langle g\rangle(\mathfrak{x})$.

Formulae (25) and (26) imply that

$$
\Phi\langle g\rangle(\mathfrak{x})=\Phi\left\langle\Psi^{\prime \prime}\langle f\rangle_{n-1}\right\rangle(\mathfrak{x}) ;
$$

from (25), (26) and (27) it follows that

(28) $\prod_{h \in \xi}$ if $h(u)=\Psi^{\prime \prime}\left\langle f_{n-1}^{\searrow}(u)\right.$ for $u \leqslant k_{3}$, then $\Phi\langle h\rangle(x)=\Phi\left\langle\Psi^{\prime \prime}\langle f\rangle_{n-1}\right\rangle(x)$, according to (16) and (17); from (28) it follows that $k_{3} \geqslant s^{\prime \prime}\left(r_{n}\right)$ and hence by (25)

$$
\text { (29) } g(u)=\Psi^{\prime \prime}\langle f\rangle_{n-1}(u) \text { for } u \leqslant s^{\prime \prime}\left(r_{n}\right) \text {. }
$$

Formulae (29) and (1) imply that

$$
\left(b-r_{n}\right) \leqslant \frac{1}{\prod_{i \leqslant s^{\prime \prime}\left(r_{n}\right)}(f(i)+1)} .
$$

The number $b$ is irrational in $f$, hence

$$
\left|b-r_{n}\right| \neq \frac{1}{\prod_{i \leqslant s^{\prime}\left(r_{n}\right)}(f(i)+1)}
$$

from (30), (31) and (18) it follows that $b \in O_{r_{n}}$.

From the compactness of the segment $[0,1]$ it follows by means of the Borel-Lebesgue theorem that for any $x \in \mathscr{N}$ and for each function $f$ there exists such a $v \in \mathscr{N}$ that the finite set of open neighbourhoods $\left\{O_{r_{0}}, \ldots, O_{r_{\nu}}\right\}$ covers the segment $[0,1]$. This statement has the form of a condition of effectivity of the minimum operation

$$
\prod_{f \in \Im} \prod_{\mathfrak{i} \in \mathcal{N V}} \Sigma_{\nu}\left\{O_{r_{0}}, \ldots, O_{r_{\nu}}\right\} \quad \text { cover }[0,1] .
$$

Now we shall prove that the relation

$$
R(f, x, v) \equiv\left\{O_{r_{0}}, \ldots, O_{r_{v}}\right\} \quad \text { cover }[0,1]
$$

is computable, and hence the minimal $v$ such that $R(f, x, v)$ is given by means of a computable functional.

The first step is to show that the functional $\Omega_{\Phi}^{*}$ is computable. From property 9 of $\S 2$ it follows that for the functional $\Phi \in \mathscr{K}$ there exists a functional $\Omega_{\Phi} \in \mathscr{K}$ such that

(34) $\prod_{g \in \mathfrak{F}}$ if $g(u)=j(u)$ for $u \leqslant \Omega_{\Phi}\langle j\rangle(\mathfrak{x})$, then $\Phi\langle j\rangle(\mathfrak{x})=\Phi\langle g\rangle(\mathfrak{x})$.

Formulae (16) and (34) imply that

$$
\Omega_{\Phi}^{*}\langle f, j\rangle(\mathfrak{x}) \leqslant \Omega_{\Phi}\langle j\rangle(\mathfrak{x}) .
$$

Hence the functional $S_{\infty}^{*}$ can be defined as follows:

$$
\begin{aligned}
\Omega_{\Phi}^{*}\langle f, j\rangle(\mathfrak{x})= & \left(\mu \varsigma \leqslant \Omega_{\Phi}\right)\left[\prod_{m \leqslant \Delta\langle f\rangle\left(\Omega_{\Phi}\right)} \quad \text { if } \quad\left\{\prod_{u \leqslant \Omega_{\Phi}} 1(m, u) \leqslant f(u)\right\}\right. \\
& \text { and } \left.\left\{\prod_{u \leqslant s} \Delta_{m}(u)=j(u)\right\}, \text { then } \Phi\left\langle\Delta_{m}\right\rangle(x)=\Phi\langle j\rangle(x)\right]
\end{aligned}
$$

where $\Omega_{\Phi}=\Omega_{\Phi}\langle j\rangle(\mathfrak{x})$.

The minimum operation snd the quantifiers contained in this definition are limited, hence $\Omega_{\Phi}^{*} \in \mathcal{K}$.

The second step is to show that the relation $r_{n}=0$ is computable. Indeed, from (5) it follows that $\Phi_{1}\langle f\rangle(n, k, x)=0$ for $x>k$. Hence according to (9) $\Psi^{\prime \prime}\langle f\rangle(n, x)=0$ for $x>\Phi_{4}\langle f\rangle(n)$. Also by (14) we have the equivalences

$$
r_{n}=0 \equiv \prod_{x \in \mathfrak{N}} \Psi^{\prime \prime}\langle f\rangle_{n-1}(x)=0 \equiv \prod_{x \leqslant \Phi_{4}\langle f\rangle(n)} \Psi^{\prime \prime}\left\langle f_{n-1}(x)=0 .\right.
$$

The relation $r_{n}=0$, defined by means of limited quantifier, is computable.

The third step is to show that the relation " $O_{r_{n}}$ intersects $O_{r_{m}}$ " is computable. Indeed the endpoints of the neighbourhoods $C_{r_{i}}$ are rationals 
and the "less than" relation between rationals is computable. Namely, according to the above mentioned property, according to which

$$
\Psi^{\prime \prime}\langle f\rangle(n, x)=0 \quad \text { for } \quad x>\Phi_{4}\langle f\rangle(n),
$$

we can limit the operation of summation in (1). Hence

$$
r_{n+\mathbf{1}}=\alpha\left(\Psi^{\prime \prime}\langle f\rangle_{n}\right)=\sum_{x=0}^{\Phi_{4}\langle f\rangle(n)} \frac{\Psi^{\prime \prime}\langle f\rangle_{n}(x)}{\prod_{i \leqslant x}(f(i)+1)} .
$$

Putting

$$
\begin{aligned}
& \Phi_{5}\langle f\rangle(n)=\sum_{x=0}^{\Phi_{4}\langle f\rangle(n)}\left\{\Psi^{\prime \prime}\langle f\rangle(n, x) \cdot \prod_{i=x+1}^{\Phi_{4}\langle f\rangle(n)}(f(i)+1)\right\}, \\
& \Phi_{6}\langle f\rangle(n)=\prod_{i \leqslant \Phi_{4}\langle f\rangle(n)}(f(i)+1)
\end{aligned}
$$

we find that

$$
r_{n+1}=\frac{\Phi_{5}\langle f>(n)}{\Phi_{6}\langle f\rangle(n)}
$$

Hence for $r_{n} \neq 0 \neq r_{m}, r_{n} \neq 1 \neq r_{m}$ it is true that

$$
O_{r_{n}} \text { intersects } O_{r_{m}}
$$

$$
\begin{aligned}
& =\frac{\Phi_{5}\langle f\rangle(n-1)}{\Phi_{6}\langle f\rangle(n-1)}+\frac{1}{\prod_{i \leqslant s^{\prime \prime}\left(r_{n}\right)}(f(i)+1)}>\frac{\Phi_{5}\langle f\rangle(m-1)}{\Phi_{6}\langle f\rangle(m-1)}+\frac{1}{\prod_{i \leqslant s^{\prime \prime}\left(r_{n}\right)}(f(i)+1)} \\
& >\frac{\Phi_{5}\langle f\rangle(n-1)}{\Phi_{6}\langle f\rangle(n-1)}-\frac{1}{\prod_{i \leqslant s^{\prime}\left(r_{n}\right)}(f(i)+1)}>\frac{\Phi_{5}\langle f\rangle(m-1)}{\Phi_{6}\langle f\rangle(m-1)}-\frac{1}{\prod_{i \leqslant s^{\prime}\left(r_{m}\right)}(f(i)+1)} .
\end{aligned}
$$

From the fact that $\Omega_{\phi}^{*} \in \mathscr{K}$ it follows that this relation is computable. If $r_{n}$ or $r_{m}$ is 0 , or 1 , then we can define the relation " $O_{r_{n}}$ intersects $O_{r_{m}}$ " in a similar way. Hence this relation is computable for any $r_{n}$ and $r_{m}$.

Now we must define the permutations of the first $n+1$ numbers: $0,1,2, \ldots, n$. Such a permutation can be represented by a number $m$ such that the following condition $\operatorname{Per}(m, n)$ is satisfied:

$$
\operatorname{Per}(m, n) \equiv m \leqslant \eta_{n}^{n} \wedge \prod_{i \leqslant n}\left\{\Delta_{m}(i) \leqslant n\right\} \wedge \prod_{i, j \leqslant n}\left\{\Delta_{m}(i)=\Delta_{m}(j) \rightarrow i=j\right\}
$$

Now it is easy to verify that

$$
\begin{gathered}
\left\{O_{r_{0}}, \ldots, O_{r_{v}}\right\} \text { cover }[0,1] \equiv\left\{\left\{v \geqslant 1 \wedge O_{r_{0}} \text { intersects } O_{r_{1}}\right\}\right. \\
\vee\left\{v>1 \wedge \sum_{m \leqslant p_{v}^{0}} \sum_{n \leqslant v} \operatorname{Per}(m, v) \wedge \prod_{i \leqslant n}\left\{r_{\Delta_{m}(i)} \neq 0 \wedge \Delta_{m}(i) \neq 0\right\}\right. \\
\wedge O_{r_{0}} \text { intersects } O_{r_{0}^{m}} \wedge \prod_{i \leqslant n}\left\{i<n \rightarrow O_{r_{i}^{m}} \text { intersects } O_{r_{i+1}^{m}}\right\} \\
\left.\wedge\left\{O_{r_{n}^{m}} \text { intersects } O_{r_{1}}\right\}\right\}
\end{gathered}
$$

where $\imath_{i}^{m}=r_{\Delta_{m}(i)}$
Hence the relation $R$ defined in (33) is computable and, according to (32), the following functional $\Phi_{\mathbf{7}}$ is computable:

$$
\Phi_{7}\langle f\rangle(x)=(\mu v)\left[\left\{O_{r_{0}}, \ldots, O_{r_{v}}\right\} \text { cover }[0,1]\right] .
$$

It is evident that for each finite set of rational numbers $\left\{r_{0}, r_{1}, \ldots, r_{n}\right\}$ such that $r_{0}=1, r_{1}=0$ the function: the least difference $d=\min \left(\left|r_{i}-r_{j}\right|\right.$ for $j, i \leqslant n$ if $r_{i} \neq r_{j}$ ) (an be expressed as a computable function. Hence the following functional $\Phi_{8}$ is computable:

$$
\begin{aligned}
& \Phi_{8}\left\langle f>(x)=(\mu s)\left[\frac{1}{\prod_{i \leqslant s}(f(i)+1)} \leqslant\right. \text { the least diameter of the }\right. \\
& \text { non empty common parts } \left.O_{r_{i}} \cap O_{r_{j}} \neq 0 \text { for } i, j \leqslant \Phi_{7}\langle f\rangle(x)\right] .
\end{aligned}
$$

Similarly the following functionals are computable:

$$
\begin{gathered}
\Phi_{9}\langle f\rangle(\mathfrak{x})=\operatorname{Max} s\left(r_{i}\right) \quad \text { for } \quad i \leqslant \Phi_{7}\langle f\rangle(\mathfrak{x}), \\
\left.\omega_{\Phi} \backslash f\right\rangle(x)=\max \left[\Phi_{8}\langle f\rangle(x), \Phi_{9}\langle f\rangle(x)\right] .
\end{gathered}
$$

The functional $\omega_{\Phi} \in \mathscr{K}$ defined above satisfies the condition (U) of the theorem. Indeed, suppose that $g, h \in 3\langle f\rangle$ and $g(u)=h(u)$ for $u \leqslant \omega_{\Phi}\langle f\rangle(\mathfrak{x})$.

From (39), (37) and (36) it follows that there exists such an $i \leqslant \Phi_{7}$ that

$$
\alpha(g), \alpha(h) \in O_{r_{i}}
$$

From (39) and (38). it follows that

$$
g(u)=h(u) \quad \text { for } \quad u \leqslant s\left(r_{i}\right) .
$$

From (15), (40) and (41) it follows that

$$
\Phi\langle g\rangle(x)=\Phi\langle h\rangle(x),
$$

which completes the proof ${ }^{10}$ ).

COROLLARY 1. The functional $v_{\Phi}$ defined by property 12 of section 2 is computable.

Proof. From the definition of the functional $v_{\Phi}$ follows the Uniformity Theorem and

$$
\nu_{\Phi}\langle f\rangle(\mathfrak{x}) \leqslant \omega_{\Phi}\langle f\rangle(\mathfrak{x}) .
$$

$\left.{ }^{10}\right)$ During the print the author has constructed a functional computable in the sense of Kleene and Mazur but not satisfying the Uniformity Theorem. 
Hence the functional $v_{\Phi}$ ean be defined as follows:

$$
\begin{aligned}
& v_{\Phi}\langle f\rangle(x)=\left(\mu s \leqslant \omega_{\Phi}\right)\left[\prod_{m_{1}, \ldots, m_{l}, n_{1}, \ldots, n_{l} \leqslant \Lambda\langle f\rangle\left(\omega_{\Phi}\right)}\right. \\
& \text { if } \prod_{u \leqslant \omega_{\Phi}}\left\{\Delta\left(m_{i}, u\right) \leqslant f(u) \wedge \Delta\left(n_{i}, u\right) \leqslant f(u)\right\} \\
& \text { and } \prod_{u \leqslant s}\left\{\Delta_{m_{i}}(u)=\Delta_{n_{l}}(u)\right\}, \\
& \text { then } \left.\Phi\left\langle\Delta_{m_{1}}, \ldots, \Delta_{m_{l}}\right\rangle(\mathfrak{x})=\Phi\left\langle\Delta_{n_{1}}, \ldots, \Delta_{n_{l}}\right\rangle(x)\right],
\end{aligned}
$$

where $\omega_{\Phi}=\omega_{\Phi}\langle f\rangle(x)$.

The minimum operation and the quantifiers contained in this definition are limited, hence $v_{\Phi}: \mathscr{K}$.

Functional InduCtion Theorem. If $\chi, \Psi, J \in \mathscr{K}$ and

$$
\begin{aligned}
\Phi\langle f\rangle_{0}(x) & =\chi\langle f\rangle(x), \\
\Phi\langle f\rangle_{n+1}(x) & =\Psi\left\langle\Phi\langle\rangle_{n}\right\rangle(x), \\
\Phi\langle f\rangle_{n}(x) & \leqslant J\langle f\rangle^{\prime}(x),
\end{aligned}
$$

for any $f \in F$ and $x \in \mathscr{N}$, then the functional

\section{is computable.}

$$
\Phi\langle f\rangle(n, x)=\Phi\langle f\rangle_{n}{ }^{\prime}(x)
$$

Proof. If $J \in \mathscr{K}$ then, according to the Corollary and to property 6 $(\S 2)$, the functional

$$
x\langle f\rangle(x)=v_{\Psi}\langle J\langle f\rangle\rangle(x)
$$

is computable and satisfies condition (d) of property 12 (§ 2). Hence, according to property $12, \Phi \in \mathscr{K}$.

\section{Applieations to the computable analysis}

There are many consequences of the Uniformity Theorem in the computable analysis. In the computable analysis we consider computable real numbers, real scquences and real functions. We assume the following definitions:

A real number a is computable $(a \in \mathscr{K})$ if there exists a function $f \in(\mathrm{Dm}$ such that for all $n \in \mathscr{N}$

$$
\left|a-\frac{f(n)}{n+1}\right|<\frac{1}{n+1}
$$

A real sequence $\left\{a_{k}\right\}$ is computable $\left(\left\{a_{k}\right\} \in \mathscr{K}\right)$ if there exists a function $f \in$ Com such that for cll $n \in \mathscr{N}$

$$
\left|a_{k}-\frac{f(k, n)}{n+1}\right|<\frac{1}{n+1} \quad \text { for any } \quad k \in \mathscr{N}
$$

A real function $\varphi$ is computable $(\varphi \in \mathscr{K})$ if there exists a functional $\Phi \in \mathscr{K}$ such that for any $f \in \mathfrak{F}$ and $a \in Z$ :

$$
\begin{gathered}
\text { if }\left|a-\frac{f(n)}{n+1}\right|<\frac{1}{n+1} \text { for any } n \in \mathscr{N}, \\
\text { then }\left|p(a)-\frac{\Phi\langle f\rangle(n)}{n+1}\right|<\frac{1}{n+1} \text { for any } n \in \mathscr{N},
\end{gathered}
$$

provided that the function $\varphi$ is defined on the numbers of the set $Z$.

Computable numbers, sequences and functions defined in such a manner are always non-negative. Functions are defined over non-negative real numbers. In order to define all computable numbers (sequences and functions), we shall replace in the above definitions the functions $f(n)(f(k, n))$ by the differences of two functions $f^{\prime}(n)-f^{\prime \prime}(n)\left(f^{\prime}(k, n)-f^{\prime \prime}(k, n)\right)$, and similarly the functional $\Phi\langle f\rangle(n)$ by the difference of two functionals $\Phi^{\prime}\langle f\rangle(n)-\Phi^{\prime \prime}\langle f\rangle(n)$. Or we can extend the notion of computability to the functions and functionals defined over the set of all integers. The following theorems can be interpreted in both manners.

In order to construct the computable analysis it seems very natural to consider the class $\mathfrak{F}^{\prime}$ of functions defined over the set $\mathscr{N}$ and assuming the values of the set $\mathfrak{N}^{\prime}$ of all integers. For these functions and for the computable functionals defined over the functions of the class $\mathfrak{F}^{\prime}$ the Uniformity Theorem has the following form:

For each functional $\Phi \in \mathscr{K}$ there exists a functional $\omega_{\Phi} \in \mathscr{K}$ such that for any $x \in \mathscr{N}$ and $f_{1}, f_{2} \in \mathfrak{F}^{\prime}$, the following condition is satisfied:

$$
\prod_{g_{1}, \ldots, g_{n}, h_{1}, \ldots, h_{n} \in \mathbf{S}^{\prime}\left\langle f_{1}, f_{\mathrm{2}}\right\rangle} \quad \text { if } \quad g_{i}(u)=h_{i}(u)
$$

for $u \leqslant \omega_{\Phi}\left\langle f_{1}, f_{2}\right\rangle(x), u \in \mathscr{N}$, then $\Phi\left\langle g_{1}, \ldots, g_{n}\right\rangle(x)=\Phi\left\langle h_{1}, \ldots, h_{n}\right\rangle(x)$, where

$$
h \in \mathcal{Z}^{\prime}\left\langle f_{1}, f_{2}\right\rangle \equiv \prod_{k \epsilon \mathfrak{V N}} f_{1}(k) \leqslant h(k) \leqslant f_{2}(k) .
$$

THEOREM 1. If $\varphi \in \mathscr{K}$ then $\varphi$ is computable in the Banach-Mazur sense. Proof. A real function $\varphi$ is computable in the Banach-Mazur sense (see Mazur [7]) provided that for each computable sequence $\left\{a_{k}\right\}$ the sequence of values of $\varphi, b_{k}=\varphi\left(a_{k}\right)$, is computable in the above-mentioned sense. Hence if $\left\{a_{k}\right\} \in \mathscr{K}$ then there exists a function $f \in \tilde{F}$ such that

$$
\left|a_{k}-\frac{f_{k}(n)}{n+1}\right|<\frac{1}{n+1} \text { for any } n \in \mathscr{N} \text {. }
$$

According to (1) we also have

$$
\left|\varphi\left(a_{k}\right)-\frac{\Phi\left\langle f_{k}\right\rangle(n)}{n+1}\right|<\frac{1}{n+1} .
$$


According to property 8 the functional $\Phi \in \mathscr{K}$ is computable in the Banach-Mazur sense. I. $e$. for $g(k, n)=\Phi\left\langle f_{k}\right\rangle(n)$ if $f \in \mathrm{Com}$, then $g \in \mathrm{Com}$. Thus

$$
\left|p\left(a_{k}\right)-\frac{g(k, n)}{n+1}\right|<\frac{1}{n+1},
$$

and also $\left\{q\left(a_{k}\right)\right\} \in \mathscr{K}$ according to the definition.

To shorten the proofs we set

$$
\mathrm{A}(a, f) \equiv \prod_{n \in \mathfrak{N}}\left|a-\frac{f(n)}{n+1}\right|<\frac{1}{n+1} .
$$

We shall apply the Uniformity Theorem in the above form to prove the following

THEOREM 2. If $\varphi \in \mathscr{K}$ then $\varphi$ is computably uniformly continuous in any segment. I. e. for $\varphi \in \mathscr{K}$ there exists a functional $\omega_{\varphi} \in \mathscr{K}$ such that if $\left.\mathrm{A}\left(a_{1}, f_{1}\right), \mathrm{A}^{\prime} a_{2}, f_{2}\right)$, then for any real $b, c$ :

(2) if $a_{1} \leqslant b, c \leqslant a_{2}$ and $|b-c|<\frac{1}{\left.\omega_{\varphi}<f_{1}, f_{2}\right\rangle(n)+1}$, then $|\varphi(b)-\varphi(c)|<\frac{2}{n+1}$.

Proof. If $\varphi \in \mathscr{K}$ then there exists a functional $\Phi \in \mathscr{K}$ such that condition (1) is satisfied. For the functional $\Phi$ there exists, according to the Unformity Theorem; a functional $\omega_{\Phi} \in \mathscr{K}$ such that condition $\left(U^{\prime}\right)$ is satisfied for any $f_{1}, f_{2} \in \widetilde{\mho}^{\prime}$. Let the function $p$ be defined in the segment $[a, b]$. We set

$$
\omega_{\Phi}\left\langle f_{1}, f_{2}\right\rangle(n)=\omega_{\Phi}\left\langle f_{1}, f_{2}\right\rangle(n) .
$$
and

Let $f_{1}, f_{2}$ be two functions such that $\mathrm{A}\left(a, f_{1}\right), \mathrm{A}\left(a, f_{2}\right)$. If $a_{1} \leqslant b, c \leqslant a_{2}$

$$
|b-c|<\frac{1}{\omega_{\varphi}\left\langle f_{1}, f_{2}\right\rangle(n)+1},
$$

then there exist two functions $g_{b}, g_{c} \in \widetilde{Y}^{\prime}$ such that

$$
\begin{gathered}
\mathrm{A}\left(b, g_{b}\right), \quad \mathrm{A}\left(c, g_{c}\right), \\
g_{b}(u)=g_{c}(u) \text { for } u \leqslant \omega_{\Phi}\left\langle f_{1}, f_{2}\right\rangle(n), \\
g_{b}, g_{c} \in \mathcal{Z}^{\prime}\left\langle f_{1}, f_{2}\right\rangle .
\end{gathered}
$$

From condition $\left(U^{\prime}\right),(5)$ and $(6)$ it follows that

$$
\Phi\left\langle g_{b}\right\rangle(n)=\Phi\left\langle g_{c}\right\rangle(n) .
$$

From (1) and (4) it follows that

$$
\left|\varphi(b)-\frac{\Phi\left\langle g_{b}\right\rangle(n)}{n+1}\right|<\frac{1}{n+1}, \quad\left|\varphi(c)-\frac{\Phi\left\langle g_{c}\right\rangle(n)}{n+1}\right|<\frac{1}{n+1} .
$$

From (7) and (8) we obtain

$$
\left.\mid \varphi^{\prime} b\right)-\varphi(e) \mid<\frac{2}{n+1} .
$$

THEOREM 3. The maximum of a function $\varphi \in \mathscr{K}$ in a segment is a co:nputable function of the endpoints of the segment. I.e. if $\varphi \in \mathscr{K}$ and $\psi(a, b)=\max _{a \leqslant c \leqslant b} \varphi(c)$, then $\Psi_{\epsilon} \mathscr{K}$.

Proof. The definition of a computable real function of two arguments is similar to the definition of a function cf cre argument. Namely, $\psi \in \mathscr{K}$ if there exists a funetional $\Psi \in \mathscr{K}$ such that

$$
\text { if } \mathrm{A}\left(a, f_{1}\right) \text { and } \mathrm{A}\left(b, f_{2}\right) \text { then } \mathrm{A}\left(\psi(a, b), \Psi\left\langle f_{1}, f_{2}\right\rangle\right) \text {. }
$$

We set

$$
\begin{gathered}
W(p \cdot f)(n)=(\mu x)\left[\left|\frac{x}{n+1}-\frac{p}{q+1}\right|<\frac{1}{n+1}\right], \\
\Psi^{\prime}\left\langle f_{1}, f_{2}\right\rangle(n)=\operatorname{Max} \Phi\left\langle W\left(x, \omega_{\Phi}\left\langle f_{1}, f_{2}\right\rangle(n)\right)>(n)\right. \\
\text { for } x \in \mathscr{N}^{\prime} \quad \text { such that } f_{1}\left(\omega_{\Phi}\left\langle f_{1}, f_{2}\right\rangle(n)\right) \leqslant x \leqslant f_{2}\left(\omega_{\Phi}\left\langle f_{1}, f_{2}\right\rangle(n)\right),
\end{gathered}
$$

where $\Phi \in \mathscr{K}$ and $\varphi$ satisfy condition (1) and $\omega_{\Phi}$ satisfies $\left(U^{\prime}\right)$. If $\Phi \in \mathscr{K}$, then $\Psi^{\prime} \in \mathcal{K}$, according to properties 2 and 5 . We shall prove that $\Psi^{\prime}$ and $\Psi$ satisfy condition (9). Indeed, let us suppose that $\mathbf{A}\left(a, f_{1}\right), \mathbf{A}\left(b, f_{2}\right)$, $a<b, \omega(n) \risingdotseq \omega_{\Phi}\left\langle f_{1}, f_{2}\right\rangle(n)$; hence

$$
\left|a-\frac{f_{1}(\omega(n))}{\omega(n)+1}\right|<\frac{1}{\omega(n)+1} ; \quad\left|b-\frac{f_{2}(\omega(n))}{\omega(n)+1}\right|<\frac{1}{\omega(n)+1} .
$$

We consider the partition of the segment $[a, b]$, by numbers of the form

where

$$
\frac{x}{\omega(n)+1}
$$

Let $d$ be such a number of the segment $[a, b]$ that

$$
\varphi(d)=\varphi(a, b)=\max _{a \leqslant c \leqslant b} \varphi(c) .
$$

Thus from (12) it follows that there exists a number $x \in \mathfrak{N}^{\prime}$, which satisfies (13) and (14), such that

$$
\left|d-\frac{x}{\omega(n)+1}\right|<\frac{1}{\omega(n)+1} .
$$


From the fact that $\omega_{\Phi}$ satisfies $\left(U^{\prime}\right)$ and $\Phi$ satisfies (1) it follows that $\varphi, \omega_{\Phi}$ satisfy (2); hence by Theorem 2, according to (2) and (16), we have

$$
\left|\varphi(d)-\varphi\left(\frac{x}{\omega(n)+1}\right)\right|<\frac{2}{n+1} .
$$

From (10) and (1) it follows that

$$
\left|\varphi\left(\frac{x}{\omega(n+1)}\right)-\frac{\Phi\langle W(x, \omega(n))\rangle(n)}{n+1}\right|<\frac{1}{n+1} .
$$

Formulae (11) and (14) imply that

$$
\Psi^{\prime}\left\langle f_{1}, f_{2}\right\rangle(n) \geqslant \Phi\langle W(x, \omega(n))\rangle(n) .
$$

Let $z \in \mathscr{N}^{\prime}$ be such a number that $z$ satisfies (14) and

$$
\Psi^{\prime}\left\langle f_{1}, f_{2}\right\rangle(n)=\Phi\langle W(z, \omega(n))\rangle(n) .
$$

From (1) and (20) it follows that

$$
\left|\varphi\left(\frac{z}{\omega(n)+1}\right)-\frac{\Psi^{\prime}\left\langle f_{1}, f_{2}\right\rangle(n)}{n+1}\right|<\frac{1}{n+1} .
$$

Formula (15) implies that

$$
\varphi(d) \geqslant \varphi\left(\frac{z}{\omega(n)+1}\right) .
$$

From (17) and (18) it follows that

$$
\left|\varphi(d)-\frac{\Phi\langle W(x, \omega(n))\rangle(n)}{n+1}\right|<\frac{3}{n+1} .
$$

From (21) it follows that

$$
\varphi\left(\frac{z}{\omega(n)+1}\right)>\frac{\Psi^{\prime}\left\langle f_{1}, f_{2}\right\rangle(n)-1}{n+1} .
$$

Formulae (19) and (24) imply that

$$
\varphi\left(\frac{z}{\omega(n)+1}\right)>\frac{\Phi\langle W(x, \omega(n))\rangle(n)-1}{n+1} .
$$

From (22) and (23) it follows that

$$
\frac{\Phi\langle W(x, \omega(n)|\rangle(n)+3}{n+1}>\varphi\left(\frac{z}{\omega(n)+1}\right) .
$$

From (25) and (26) we obtain

$$
\left|\varphi\left(\frac{z}{\omega(n)+1}\right)-\frac{\Phi\langle W(x, \omega(n))\rangle(n)}{n+1}\right|<\frac{3}{n+1} .
$$

Formulae (21) and (27) give

$$
\left|\frac{\Psi^{\prime}\left\langle f_{1}, f_{2}\right\rangle(n)}{n+1}-\frac{\Phi\langle W(x, \omega(n)|\rangle(n)}{n+1}\right|<\frac{4}{n+1} .
$$

From (28) and (23) it follorss that

Now we put

$$
\left|\varphi(d)-\frac{\Psi^{\prime}\left\langle f_{1}, f_{2}\right\rangle(n)}{n+1}\right|<\frac{7}{n+1} .
$$

Formula (29) implies that

$$
\Psi\left\langle f_{1}, f_{2}\right\rangle(n)=\left[\frac{\Psi^{\prime}\left\langle f_{1}, f_{2}\right\rangle(14 n+13)+\tau}{14}\right] .
$$

$$
\begin{array}{ll}
\text { (31) } & \left|\varphi(d)-\frac{\Psi^{\prime}\left\langle f_{1}, f_{2}\right\rangle(14 n+13)}{14 n+14}\right|<\frac{7}{14 n+14}=\frac{1}{2(n+1)} . \\
\text { Hence } & \frac{\Psi^{\prime}\left\langle f_{1}, f_{2}\right\rangle(14 n+13)}{14(n+1)}+\frac{1}{2(n+1)}<\varphi(d)+\frac{1}{n+1} \\
\text { and } & {\left[\frac{\Psi^{\prime}\left\langle f_{1}, f_{2}\right\rangle(14 n+13)}{14}+\frac{1}{2}\right] \leqslant \frac{\Psi^{\prime}\left\langle f_{1}, f_{2}\right\rangle(14 n+13)}{14}+\frac{1}{2}} \\
\text { (32) } & <(n+1) \varphi(d)+1 .
\end{array}
$$

On the other hand, from (31) it follows that

hence

$$
\varphi(d)-\frac{1}{n+1}<\frac{\Psi^{\prime}\left\langle f_{1}, f_{2}\right\rangle(14 n+13)}{14(n+1)}-\frac{1}{2(n+1)} ;
$$

$$
\begin{gathered}
(n+1) \varphi(d)-1<\frac{\Psi^{\prime}\left\langle f_{1}, f_{2}\right\rangle(14 n+13)}{14}-\frac{1}{2} \\
<\left[\frac{\Psi^{\prime}\left\langle f_{1}, f_{2}\right\rangle(14 n+13)}{14}+\frac{1}{2}\right] .
\end{gathered}
$$

From (30), (32) and (33) it follows that

$$
(n+1) \varphi(d)-1<\Psi\left\langle f_{1}, f_{2}\right\rangle(n)<(n+1) \varphi(d)+1 .
$$

This means that

$$
\left|\varphi(d)-\frac{\Psi\left\langle f_{1}, f_{2}\right\rangle(n)}{n+1}\right|<\frac{1}{n+1},
$$

which completes the proof. 
THEOREM 4. If the function $\varphi \in \mathscr{K}$ is defined over the segment $[a, b]$, $\mathbf{A}\left(a, f_{1}\right), \mathbf{A}\left(b, f_{2}\right)$ and $d$ is the unique number of the segment $[a, b]$ such that $\varphi(d)=\max \varphi(c)$, then $d$ is computable in $f_{1}, f_{2}$.

Proof. We consider the partition of the segment $[a, b]$ by numbers of the form

$$
\frac{x}{\omega_{1}(n)+1}, \quad \text { where } \quad \omega_{1}(n)=\omega_{\Phi}\left\langle f_{1}, f_{2}\right\rangle(n)+n+1
$$

and

$$
f_{1}\left(\omega_{1}(n)\right) \leqslant x \leqslant f_{2}\left(\omega_{1}(n)\right)
$$

From Theorem 2 it follows that for any $d \epsilon[a, b]$ there exists an $x \in \mathscr{N}^{\prime}$ which satisfies (35) and

$$
\left|\varphi(d)-\varphi\left(\frac{x}{\omega_{1}(n)+1}\right)\right|<\frac{2}{n+1} .
$$

Suppose that $\varphi(d)=\max \varphi(c)$; hence according to Theorem 3 there exists a functional $\Psi \in \mathscr{K}$ such that $\mathrm{A}\left(\varphi(d), \Psi\left\langle f_{1}, f_{2}\right\rangle\right)$, hence according to $(36)$

$$
\left|\frac{\Psi\left\langle f_{1}, f_{2}\right\rangle(n)}{n+1}-\varphi\left(\frac{x}{\omega_{1}(n)+1}\right)\right|<\frac{3}{n+1} .
$$

On the other hand, from (1) it follows that

$$
\mathrm{A}\left(\varphi\left(\frac{x}{\omega_{1}(n)+1}\right), \Phi\left\langle W\left(x, \omega_{1}(n)\right)\right\rangle\right) .
$$

Thus according to (37) we obtain the following condition:

$$
\begin{gathered}
\prod_{n \in \mathscr{N}} \sum_{x \in \mathscr{N}} x \text { satisfies }(35) \\
\text { and } \quad\left|\frac{\Psi\left\langle f_{1}, f_{2}\right\rangle(n)}{n+1}-\frac{\Phi\left\langle W\left(x, \omega_{1}(n)\right)\right\rangle(n)}{n+1}\right|<\frac{4}{n+1} .
\end{gathered}
$$

The following functional is also computable:

$$
\begin{gathered}
\xi(n)=(\mu x)\left[f_{1}\left(\omega_{1}(n)\right) \leqslant x \leqslant f_{2}\left(\omega_{1}(n)\right)\right. \\
\left.\wedge\left|\Psi\left\langle f_{1}, f_{2}\right\rangle(n)-\Phi<W\left(x, \omega_{1}(n)\right)\right\rangle(n) \mid<4\right] .
\end{gathered}
$$

If $\varphi(d)$ is the unique maximum of the function $\varphi$ in the segment $[a, b]$, then the following condition is satisfied:
$(40)$

$$
\begin{aligned}
& \prod_{k \in \mathfrak{N} N} \sum_{n \in \mathscr{N}} \omega_{1}(n)+1>6(k+1) \text { and } \prod_{y, x \in \mathscr{F}} \\
& \quad \text { if } f_{1}\left(\omega_{1}(n)\right) \leqslant x, y \leqslant f_{2}\left(\omega_{1}(n)\right) \\
& \text { and }\left|\Psi\left\langle f_{1}, f_{2}\right\rangle(n)-\Phi\left\langle W\left(x, \omega_{1}(n)\right)\right\rangle(n)\right|<4 \\
& \text { and }\left|\Psi\left\langle f_{1}, f_{2}\right\rangle(n)-\Phi<W\left(y, \omega_{1}(n)\right)\right\rangle(n) \mid<4, \\
& \text { then } \frac{|x-y|}{\omega_{1}(n)+1}<\frac{1}{6(k+1)} .
\end{aligned}
$$

Let $\zeta(k)=$ the smallest $n$ such that $k$ and $n$ satisfy condition (40). The function $\zeta$ is computable in $f_{1}, f_{2}$ under the suppositions of the theorem. Now we put

$$
g(k)=(\mu z)\left[\left|\frac{z}{k+1}-\frac{\xi(\zeta(k))}{\omega_{1}(\zeta(k))+1}\right|<\frac{2}{3(k+1)}\right] .
$$

We shall prove that $A(d, g)$. Indeed, from (41) we have

$$
\left|\frac{g(k)}{k+1}-\frac{\xi(\zeta(k))}{\omega_{1}(\zeta(k))+1}\right|<\frac{2}{3(k+1)} .
$$

From (34) and (35) it follows that there exists such an $x \in \mathfrak{N}^{\prime}$ that

$$
\begin{gathered}
f_{1}\left(\omega_{1}(\zeta(k))\right) \leqslant x \leqslant f_{2}\left(\omega_{1}(\zeta(k))\right), \\
\left|\frac{x}{\omega_{1}(\zeta(k))+1}-d\right|<\frac{1}{\omega_{1}(\zeta(k))+1} .
\end{gathered}
$$

Hence according to (37), (38) and Theorem 3

$$
\left|\Psi\left\langle f_{1}, f_{2}\right\rangle(\zeta(k))-\Phi\left\langle W\left(x, \omega_{1}(\zeta(k))\right)\right\rangle\langle\zeta(k))\right|<4 .
$$

From (41), (39), (40), (43) and (45) it follows that

$$
\begin{aligned}
& \omega_{1}(\zeta(k))+1>6(k+1), \\
& \frac{|x-\xi(\zeta(k))|}{\omega_{1}(\zeta(k))+1}<\frac{1}{6(k+1)} .
\end{aligned}
$$

Formulae (46), (44) and (47) imply that

$$
\left|\frac{\xi(\zeta(k))}{\omega_{1}(\zeta(k))+1}-d\right|<\frac{1}{3(k+1)} .
$$


From (42) and (48) we obtain

$$
\left|\frac{g(k)}{k+1}-d\right|<\frac{1}{k+1}
$$

which completes the proof.

THEOREM 5. The computable real numbers constitute the field of numbers algebraically closed in the field of real numbers.

Proof. It is very easy to verify that the functions $+,-, \cdot,:$, are computable functions of two arguments. Hence the set of computable numbers is a field of numbers, and any polynomial $\pi(x)$ with computable coefficients is a computable function. Similarly the function $-|\pi(x)|$ is also computable and satisfies the conditions of Theorem 4 . Hence $-|\pi(x)|$ assumes all maxima in computable points. But $-|\pi(x)|$ assumes a maximum if and only if $\pi(x)=0$; thus the real roots of the polynomial $\pi$ are computable $\left.{ }^{11}\right)$.

THEOREM 6. If $\varphi \in \mathscr{K}$ and $\varphi$ is strictly monotonic in the segment $[a, b]$ and $\mathrm{A}\left(a, f_{1}\right), \mathrm{A}\left(b, f_{2}\right)$, then the function $\varphi^{-1}$ is computable in $f_{1}, f_{2}$.

Proof. The proof is similar to the proof of Theorem 4. First we consider the partition of the segment $[a, b]$ by numbers satisfying (34) and (35). Similarly to (39) we set

$\left(39^{\prime}\right) \xi\langle g\rangle(n)=(\mu x)\left[f_{1}\left(\omega_{1}(n)\right) \leqslant x \leqslant f_{2}\left(\omega_{1}(n)\right) \wedge\left|g(n)-\Phi<W\left(x, \omega_{1}(n)\right)\right\rangle(n) \mid<4\right] ;$ the functional $\xi$ is computable because the operation of minimum is limited. From the fact that the function $\varphi$ is strictly monotonic it follows that:

$\left(40^{\prime}\right) \quad \prod_{g \in \mathcal{J}^{\prime}} \prod_{k \in \mathcal{S N}} \sum_{n \in \mathcal{V} \mathcal{V}} \omega_{1}(n)+1>6(k+1)$ and $\prod_{x, y \in \mathcal{V N}}$

if $\quad f_{1}\left(\omega_{1}(n)\right) \leqslant x, y \leqslant f_{2}\left(\omega_{1}(n)\right) \quad$ and $\quad\left|g(n)-\Phi<W\left(x, \omega_{1}(n)\right)>(n)\right|<4$ and $\left|g(n)-\Phi<W\left(y, \omega_{1}(n)\right)\right\rangle(n) \mid<4$, then $\left|\frac{x-y}{\omega_{1}(n)+1}\right|<\frac{1}{6(k+1)}$.

Let $\zeta\langle g\rangle(k)$ be the smallest $n \in \mathscr{V}$ such that $g, k, n$ satisfy condition $\left(40^{\prime}\right)$. Thus $\zeta \epsilon \mathscr{K}$.

Now we put

$$
\Phi^{-1}\langle g\rangle(k)=(\mu x)\left[\left|\frac{x}{k+1}-\frac{\xi\langle g\rangle}{\omega_{1}(\zeta\langle g\rangle(k))+1}\right|\left\langle\frac{\zeta\langle g\rangle(k))}{3(k+1)}\right] .\right.
$$

We shall prove that if $\mathbf{A}(c, g)$, then $\mathbf{A}\left(\varphi^{-1}(c), \Phi^{-1}\langle g\rangle\right)$ for $a \leqslant \varphi^{-1}(c) \leqslant b$. Indeed, suppose that

$$
\left(42^{\prime}\right) \quad \mathrm{A}(c, g), \quad a \leqslant \varphi^{-1}(c) \leqslant b .
$$

II) Another proof of Theorem 5 is in Mazur [7].
Hence there exists.such an $x \in \mathscr{N}^{\prime}$ that

$$
\begin{gathered}
f_{1}\left(\omega_{1}(\zeta\langle g\rangle(k))\right) \leqslant x \leqslant f_{2}\left(\omega_{1}(\zeta\langle g\rangle(k))\right), \\
\left|\varphi^{-1}(c)-\frac{x}{\omega_{1}(\zeta\langle g\rangle(k))+1}\right|<\frac{1}{\omega_{1}(\zeta\langle g\rangle(k))+1} .
\end{gathered}
$$

From $\left(44^{\prime}\right)$, by Theorem 2, it follows that

$$
\left|c-\varphi\left(\frac{x}{\omega_{1}(\zeta\langle g\rangle(k))+1}\right)\right|<\frac{2}{\zeta\langle g\rangle(k)+1} .
$$

Formulae $\left(42^{\prime}\right),\left(45^{\prime}\right)$ and (1) imply that

$\left(46^{\prime}\right) \quad\left|g(\zeta\langle g\rangle(k))-\Phi\left\langle W\left(x, \omega_{1}(\zeta\langle g\rangle(k))\right)\right\rangle\langle\zeta\langle g\rangle(k))\right|<4$.

On the other hand, from $\left(39^{\prime}\right)$ it follows that

$\left(48^{\prime}\right)\left|g(\zeta\langle g\rangle(k))-\Phi\left\langle W\left(\xi\langle g\rangle(\zeta\langle g\rangle(k)), \omega_{1}(\zeta\langle g\rangle\langle k))\right)\right\rangle\langle\zeta\langle g\rangle(k))\right|<4$,

$$
\omega_{1}(\zeta\langle g\rangle(k))+1>6(k+1) \text {. }
$$

From $\left(40^{\prime}\right),\left(43^{\prime}\right),\left(46^{\prime}\right),\left(47^{\prime}\right)$ and $\left(48^{\prime}\right)$ it follows that

$$
\left|\frac{x-\xi\langle g\rangle\langle\zeta\langle g\rangle(k))}{\omega_{1}(\zeta\langle g\rangle(k))+1}\right|<\frac{1}{6(k+1)} .
$$

Formulae $\left(44^{\prime}\right),\left(49^{\prime}\right)$ and $\left(50^{\prime}\right)$ imply that

$$
\left|\varphi^{-1}(c)-\frac{\xi\langle g\rangle(\zeta\langle g\rangle(k))}{\omega_{1}(\zeta\langle g\rangle(k))+1}\right|<\frac{1}{3(k+1)} .
$$

From $\left(41^{\prime}\right)$ and $\left(51^{\prime}\right)$ we obtain

$$
\left|\varphi^{-1}(c)-\frac{\Phi^{-1}\langle g\rangle(k)}{k+1}\right|<\frac{1}{k+1}
$$

which completes the proof.

THEOREM 7. There exists a function $\chi \in \mathscr{K}$ which is not differentiable at any point.

Proof. Let us put

(1) $\varphi(a)=$ the smaller of two numbers: $\{a-[a],[a+1]-a\}$,

$$
\chi(a)=\sum_{n=0}^{\infty} \frac{\varphi\left(10^{n+1} \cdot a\right)}{10^{n+1}} .
$$


Both functions, $\varphi$ and $\chi$, are computable.. Indeed, let us consider the computable functionals

$$
\begin{gathered}
\Phi_{1}\langle f\rangle(n)=(\mu x)[|f(n)-(x+1) \cdot(n+1)| \\
\geqslant|f(n)-x \cdot(n+1)| \leqslant|f(n)-(x-1) \cdot(n+1)|], \\
\Phi\langle f\rangle(n)=\left|\Phi_{1}\langle f\rangle(n) \cdot(n+1)-f(n)\right| .
\end{gathered}
$$

We shall prove that if $\mathrm{A}(a, f)$, then $\mathrm{A}(\varphi(a), \Phi\langle f\rangle)$.

Let us suppose that

$$
\left|\frac{f(n)}{n+1}-a\right|<\frac{1}{n+1} \quad \text { for any } \quad n \in \mathscr{N} \text {. }
$$

From (3) and (5) it follows that

$$
\begin{aligned}
& \text { if } \frac{f(n)}{n+1}>[a]+\frac{1}{2}, \text { then } \Phi_{1}\langle f\rangle(n)=[a]+1, \\
& \text { if } \frac{f(n)}{n+1} \leqslant[a]+\frac{1}{2}, \text { then } \Phi_{1}\langle f\rangle(n)=[a] .
\end{aligned}
$$

Hence from (4) and (6) we obtain

$$
\begin{aligned}
& \frac{\Phi\langle f\rangle(n)}{n+1}=\text { the smaller of two number's } \\
& \quad\left\{\left|\frac{f(n)}{n+1}-[a]\right|,\left|[a+1]-\frac{f(n)}{n+1}\right|\right\} .
\end{aligned}
$$

Now let us distinguish 6 cases:

$$
\text { A. } a=\frac{f(n)}{n+1} \text {. }
$$

From (7) and (1) it follows that $\frac{\Phi\langle f\rangle(n)}{n+1}=\varphi(a)$.

$$
\text { B. } a=[a]+\frac{1}{2} \text {. }
$$

If $n$ is odd, then $a=\frac{f(n)}{n+1}, i . e$. the case $A$, if $n$ is even, then

$$
\left|\frac{f(n)}{n+1}-a\right|=\frac{1}{2(n+1)} \quad \text { and } \quad\left|\frac{\Phi\langle f\rangle(n)}{n+1}-\varphi(a)\right|=\frac{1}{2(n+1)} .
$$

C. $a<[a]+\frac{1}{2}<\frac{f(n)}{n+1} \quad\left(\right.$ or $\left.a>[a]+\frac{1}{2}>\frac{f(n)}{n+1}\right)$.

$$
\begin{array}{rlrl}
\text { Hence } & \left|\frac{f(n)}{n+1}-[a]+\frac{1}{2}\right|=\frac{1}{2(n+1)} & \text { and } & \left|a-[a]+\frac{1}{2}\right|<\frac{1}{2(n+1)}, \\
\frac{\Phi\langle f\rangle(n)}{n+1}+\frac{1}{2(n+1)}=\frac{1}{2}, & \text { thus } & \left|\varphi(a)-\frac{\Phi\langle f\rangle(n)}{n+1}\right|<\frac{1}{2(n+1)} .
\end{array}
$$

D. $a<\frac{f(n)}{n+1} \leqslant[a]+\frac{1}{2} \quad$ (or $\left.[a] \leqslant \frac{f(n)}{n+1}<a<[a]+\frac{1}{2}\right)$.

Hence $\quad \frac{f(n)}{n+1}=[a]+\frac{\Phi\langle f\rangle(n)}{n+1}$ and $\quad\left|\frac{\Phi\langle f\rangle(n)}{n+1}-\varphi(a)\right|<\frac{1}{n+1}$.

E. $a>\frac{f(n)}{n+1} \geqslant[a]+\frac{1}{2} \quad$ (or $\left.[a]+1 \geqslant \frac{f(n)}{n+1}>a>[a]+\frac{1}{2}\right)$.

Hence $\quad \frac{f(n)}{n+1}=[a+1]-\frac{\Phi\langle f\rangle(n)}{n+1} \quad$ and $\quad\left|\frac{\Phi\langle f\rangle(n)}{n+1}-\varphi(a)\right|<\frac{1}{n+1}$.

F. $\frac{f(n)}{n+1}<[a]=a$.

Hence $\quad\left|\frac{f(n)}{n+1}-[a]\right|=\frac{1}{2(n+1)} \quad$ and $\quad \frac{\Phi \nvdash f>(n)}{n+1}=\frac{1}{2(n+1)}$,

then $\left|\varphi(a)-\frac{\Phi\langle f\rangle(n)}{n+1}\right|=\frac{1}{2(n+1)}$.

From (1), (5), (6) and (7) we also find that in any case

$$
\left|\frac{\Phi\langle f\rangle(n)}{n+1}-\varphi(a)\right|<\frac{1}{n+1}
$$

which proves that $\varphi \in \mathscr{K}$.

It is evident that if $\varphi \in \mathscr{K}$ then $\chi \in \mathscr{K}$, because $\varphi\left(10^{n+1} \cdot a\right)<1$; thus it is easy to compute the value of $\chi(a)$ with any estimation. The function $\chi$ is well known to be non-differentiable at any point.

There remain some interesting problems concerning the computable real functions. $E . g$. whether the maximum of a computable function is always obtained in a computable point, whether the derived function of a differentiable computable function is computable, a similar problem concerning integration and others ${ }^{12}$ ).

$\left.{ }^{13}\right)$ During the print $\mathrm{S}$. Mazur and the author have remarked that the class $\mathscr{K}$ of computable real functions of $\S 4$ can be defined without use of computable functionals in a manner similar to the definition of recursive real function formulated by E. Specker in [14]. Namely $\varphi \in \mathscr{K}$ if and only if there exist the functions $f, g, h, j \in \mathrm{Som}$ such that:

1. $\varphi$ is continuous;

2. $\varphi(p / q)=\lim _{n \rightarrow \infty}(h(p, q, n) / j(p, q, n))$;

3. if $n, m>f(k)$, then $|h(p, q, n) / j(p, q, n)-h(p, q, m) / j(p, q, m)|<1 /(k+1)$;

4. if $t / u \leqslant p / q<r / s \leqslant v / w$, and $|p / q-r / s|<1 / g(t, u, v, w, k)$, then $\mid h(p, q, n) / j(p, q, n)-$ $-h(r, s, n) / j(r, s, n) \mid<1 /(k+1)$ 


\section{References}

[1] A. Grzegorczyk, Some classes of recursive functions, Rozprawy Matema. tyczne 4, Warszawa 1953.

[2] - Elementarily definable analysis, Fundamenta Mathematicae 41 (1954), p. $311-338$.

[3] S. C. Kleene, Recursive predicates and quantifiers, Trans. Am. Math. Soc. 53 (1943), p. 41-73.

[4] - On the interpretation of intuitionistic number theory, Journ. Symb. Log. 10 (1945), p. 109-124.

[5] - Introduction to Metamathematics, Amsterdam 1952.

[6] А. А. Марков, о представлении рекурсивных функиий, Ивв. Акад. Наук СССР, серия мат. 13 (1949), p. 417-424.

[7] S. Mazur, Introduction to the computable analysis, to appear.

[8] A. Mostowski, On definable sets of positive integers, Fundamenta Mathematicae 34 (1947), p. 81-112.

[9] D. Nelson, Recursive functions and intuitionistic number theory, Trans. Am. Math. Soc. 61 (1947), p. 307-368.

[10] R. Peter, Über den Zusammenhang der verschiedenen Begriffe der rekursiven Funktion, Math. Annalen 110 (1935), p. 612-632.

[11] - Rekursive Funktionen, Budapest 1951.

[12] E. L. Post, Recursively enumerable sets of positive integers and their decision problems, Bull. Am. Math. Soc. 50 (1944), p. 284-316.

[13] J. Robinson, General recursive functions, Proc. Amer. Math. Soc. 1 (1950), p. 703-718.

[14] E. Specker, Nicht konstruktiv beweisbare Sätze der Analysis, Journ. Symb. Log. 14 (1949), p. 145-158.

\section{INSTYTUT MATEMATYCZNY POLSKIEJ AKADEMII NAUK}

MATHEMATICAL INSTITUTE OF THE POLISH ACADEMY OF SCIENCES

Reģu par la Rédaction le 21. 7. 1954

\section{Continuous mappings of a certain family}

by

\section{J. de Groot (Laren, Holland)}

1. We shall prove as a main result, using the axiom of choice ( $c$ denotes the potency of the system of real numbers):

THEOREM I. There exists a family of $2^{\mathbf{c}}$ subsets of the plane such that no set of the family can be mapped continuously (degenerate maps excepted ${ }^{1}$ )) in (or on) another set of the family.

This theorem is known if the "continuous maps in" are replaced by "topological maps in" or "continuous maps on" or even "continuous maps in, with c image-points" ${ }^{2}$ ). While these theorems are true and have been proved in any complete separable space, this is clearly not the case with theorem $I$. In the discontinuum of Cantor $D$ for example any subset of $D$ having dimension 0 may be mapped continuously and non-degenerately in any subset of $D$, consisting of more than one point. Moreover it is clear that the family of theorem I must in any case consist of connected sets containing no arcs. On the other hand the other theorems are true both for (suitably selected) families of connected or not connected sets and (except in one case) for families containing arcs or no arcs.

From these considerations one might expect that a proof of theorem I might be rather difficult. This is, however, not the case if we add some topological features to the proof of a general set-theoretic theorem of Kuratowski ${ }^{3}$ ). Our proof parallels therefore to a great extent the proof of Kuratowski, which enables him to establish the mentioned theorem in the case of "continuous maps in with $c$ image-points". On the other hand this proof is only an existence-proof using the axiom of choice. It is easy e.g. to define effectively two sets neither of which can be map-

1) A map is called degenerate if the image consists of one point. Henceforth all maps considered will be non-degenerate.

$\left.{ }^{2}\right)$ Comp. C. Kuratowski, Topologie I, Warszawa 1952, p. 330-341. Associated with the theorems stated are the names of Kuratowski, Sierpinski, Lindenbaum, Waraszkiewicz.

3) 0. c., p. 332, Théorème auxiliaire. 